\title{
A database on flash flood events in Campania, southern Italy, with an evaluation of their spatial and temporal distribution
}

\author{
Carmela Vennari $^{1,2}$, Mario Parise ${ }^{2}$, Nicoletta Santangelo ${ }^{1}$, and Antonio Santo ${ }^{3}$ \\ ${ }^{1}$ Department of Earth Sciences, University of Naples Federico II, Largo San Marcellino 10, Naples, Italy \\ ${ }^{2}$ Italian National Research Council, Research Institute for Geo-hydrological Protection, Bari, Italy \\ ${ }^{3}$ Department of Hydraulic, Geotechnical and Environmental Engineering, Applied Geology Division, \\ University of Naples Federico II, Naples, Italy
}

Correspondence to: Carmela Vennari (c.vennari@ba.irpi.cnr.it)

Received: 30 December 2015 - Published in Nat. Hazards Earth Syst. Sci. Discuss.: 19 January 2016

Revised: 8 November 2016 - Accepted: 9 November 2016 - Published: 29 November 2016

\begin{abstract}
This study presents an historical database of flash flood events in the Campania region of southern Italy. The study focuses on small catchments characterized by intermittent flow, generally occurring during and after heavy rainstorms, which can be hydrologically defined as small Mediterranean catchments. As the outlet zones of these catchments (consisting mainly of alluvial fans or fan deltas) are highly urbanized in Campania, the population living in the delivery areas is exposed to high risk. Detailed scrutiny and critical analysis of the existing literature, and of the data inventory available, allowed us to build a robust database consisting of about 500 events from 1540 to 2015, which is continuously updated. Since this study is the first step of a longer project to perform a hazard analysis, information about time and site of occurrence is known for all events. As for the hazard analysis envisaged, collecting information about past events could provide information on future events, in terms of damage and also spatial and temporal occurrence. After introducing the issue of flash floods in Italy we then describe the geological and geomorphological settings of the study area. The database is then presented, illustrating the methodology used in collecting information and its general structure. The collected data are then discussed and the statistical data analysis presented.
\end{abstract}

\section{Introduction}

Italy as a country is highly exposed to a variety of geological hazards, whose occurrence reaps heavy casualties every year. In such a context, geohydrological disasters, including all types of slope movements and floods, are undoubtedly among the most frequent, and probably those causing most impact on the built environment. Assessment of the risk related to landslides and floods in Italy, covering the time span from 1850 to 2008 , shows that many regions are seriously affected by such geohydrological disasters, with the largest landslide risk in Trentino Alto Adige and Campania and the highest flood risk in Piedmont and Sicily (Salvati et al., 2010). However, discriminating among these different processes, especially in regard to past events, is a difficult task due to the lack of specific terminology and/or to very generic descriptions of the phenomena and their effects (Guzzetti and Tonelli, 2004). This may, as a direct consequence, lead to great uncertainty in the reconstruction of the flood history of a region.

A flash flood is a flood caused by heavy or excessive rainfall in a short period of time. Such floods are localized hydrological phenomena, occurring in small catchments of a few to a few hundred square kilometers, with response times typically being a few hours or less (Borga et al., 2007). Flash floods are typical of small Mediterranean catchments (SMCs) that have three main features: limited water resources, dry summers, and high-intensity rainfall events (Merheb et al., 2016). The Mediterranean climate is associated with intense rainstorms; convective thunderstorms are frequently less than $10-14 \mathrm{~km}$ in diameter and result in highly concentrated local rainfall events varying considerably both spatially and temporally (Perrin et al., 2009). In such contexts, after heavy rains, the main stream may trans- 
port down-valley mixtures of water and sediment in varying proportions, which play an important role in the behavior and hazard of the resulting flows. Three types of flows can be differentiated on the basis of the different amount of sediment and of the flow behavior: water flow, hyperconcentrated flow, and debris flow (Pierson and Costa, 1987; Costa, 1988; Komar, 1988; Iverson and Vallance, 2001; Pierson, 2005). During a flash flood the sediments captured by the stream and transported down valley generally derive from the bed and banks of the torrent and may eventually contribute to build alluvial fans or fan deltas. Another important aspect is that in small catchments a substantial difference of magnitude exists between low water periods, with a seasonal lack of surface water within ephemeral streams, and flood events with the power to make substantial changes to riverbed topography (Kirkham et al., 2000; Coe et al., 2003).

Flash floods are a frequent natural hazard in many parts of Europe (Montz and Gruntfest, 2002; Gaume et al., 2009, 2014; Marchi et al., 2010), including Italy. Due to the particular orography and climate of the country, they occur in many different settings, from mountain valleys (Crosta and Frattini, 2004; Tropeano and Turconi, 2004; Gaume et al., 2009) to coastal and inland plains (Esposito et al., 2011; Santangelo et al., 2011; Porfido et al., 2013), in volcanic areas (Alessio et al., 2013), and in semiarid and/or karst environments (Parise, 2003; Cossu et al., 2007; Delle Rose and Parise, 2010). Flash floods generally occur in ungauged watersheds where the lack of information on precipitation and discharge is significant due to the lack of spatially well-distributed rain or flow data. Hence they often remain poorly documented phenomena (Gaume et al., 2009; Ruiz-Villanueva et al., 2010) and, despite their widespread occurrence, they are often described together with landslides and floods in alluvial plains, making it no easy task to distinguish the various processes occurring from documents and reports. This holds especially when examining events occurring at various times in the past in which recorded damage is generally attributed to landslides.

Whilst in the Italian Alps, due to their high frequency, flash floods in small basins have been the subject of detailed studies for many decades (Crosta and Frattini, 2004; Sosio et al., 2007; Carrara et al., 2008; Simoni et al., 2011; Arattano et al., 2012, 2015; Marchi and Tecca, 2013; Berti and Simoni, 2014; Blahut et al., 2014; Tiranti et al., 2014), in the Apennines they have typically been analyzed with less attention (Sorriso Valvo et al., 1998; Zanchetta et al., 2004a; Santo et al., 2002, 2012, 2015; Garfî et al., 2007; Cascini et al., 2008a; Santangelo et al., 2011, 2012; Alessio et al, 2013; Antronico et al., 2015a, b; Scorpio et al., 2016).

The study area of this paper is situated in Campania, in the Southern Apennines of Italy, a region which in recent decades has been affected by severe flash floods with serious damage and fatalities (Calcaterra et al., 2000, 2003; Santo et al., 2002, 2012, 2015; Del Prete and Mele, 2006; Santangelo et al., 2006, 2011, 2012; Chirico et al., 2012; Alessio et al., 2013). The focus of the study consisted of small catch-

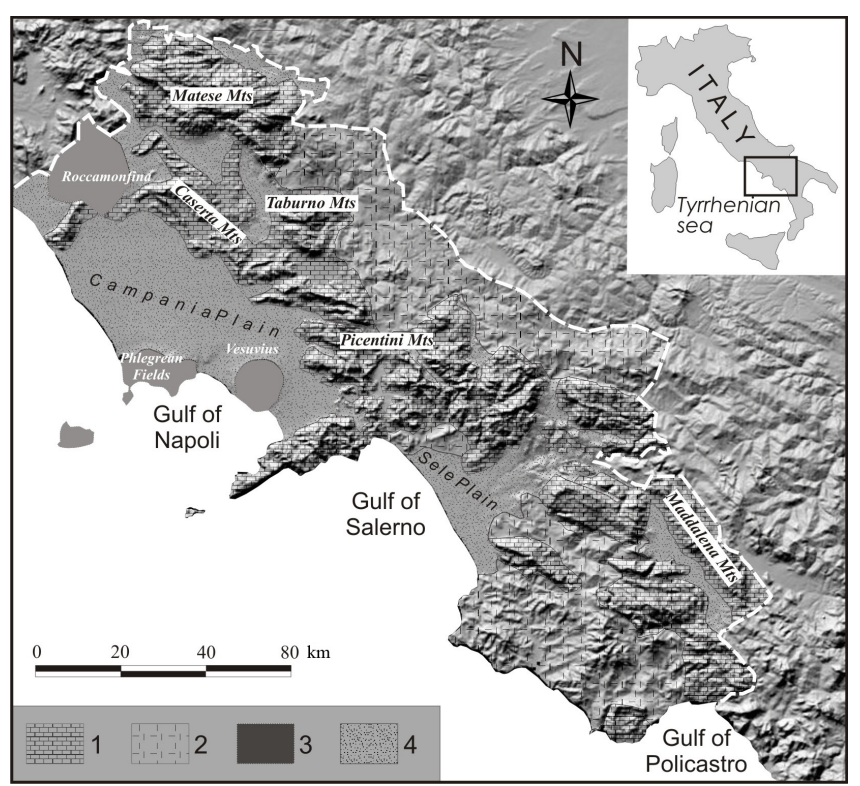

Figure 1. Location and geological setting of the study area. Key: (1) Mesozoic carbonate massifs; (2) Cenozoic hilly terrigenous areas; (3) Quaternary volcanic areas; (4) Quaternary intermountain catchments and coastal plains. The broken line indicates the boundaries of Campania.

ments with intermittent flow, generally occurring during and after heavy rainstorms, which can be hydrologically defined as SMCs. All these basins are smaller than $10 \mathrm{~km}^{2}$, are characterized by low concentration times (from $30 \mathrm{~min}$ to several hours; Santo et al., 2002; Santangelo et al., 2012; Scorpio et al., 2016), and are highly prone to flash flood events. Aiming at collecting all the available information on floods occurring in such catchments, we consulted and scrutinized a variety of sources and scientific papers, with a view to building a reliable catalogue of these events.

As the first step in the process of hazard evaluation, we compiled the database with the following main aims: (i) to identify over the whole region the areas most susceptible to flash floods and (ii) to discriminate whenever possible, in the available literature, flash floods in small catchments from floods in alluvial plains and from gravity processes, such as rapid earth or debris flow (Del Prete et al., 1998; Crosta and Dal Negro, 2003; Guadagno et al., 2003, 2005; Revellino et al., 2004; Zanchetta et al., 2004b; Di Crescenzo and Santo, 2005; Cascini et al., 2008b). Our database is not aimed at a hydrological characterization of the study areas due to lack of hydrological data, especially as concerns the historical events. Nevertheless, given the lack of similar catalogues for the Southern Apennines of Italy, it might be useful in different ways for land use and civil protection planning. In detail, it may help in the selection of sites where monitoring procedures and/or prevention and mitigation works need to be adopted. In this perspective the main users of such data will be local administrators and the civil protection agency. 


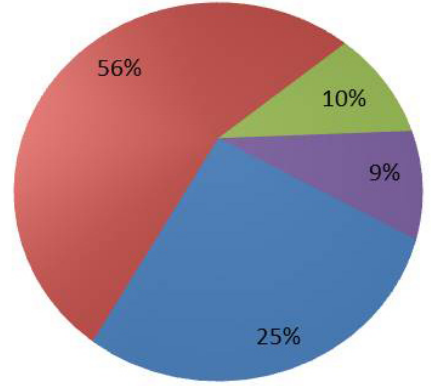

- Flood events in alluvial plain context - Flash floods in torrential stream basin - Landslides

- Mixed and doubtful cases

Figure 2. Event types collected while constructing the database.

\section{Study area}

The region of Campania in southern Italy extends from the Tyrrhenian Sea to the Southern Apennine Chain, covering about $13500 \mathrm{~km}^{2}$ (Fig. 1). The orographic setting is characterized by the presence of a central mountain ridge made up mainly of Mesozoic carbonates, elongated for more than $200 \mathrm{~km}$ in a NW-SE direction, with maximum peaks reaching $2000 \mathrm{~m}$ a.s.l. (above sea level). On the western side the chain is bounded by a deep (up to $2 \mathrm{~km}$ ) coastal graben originated by Plio-Quaternary extensional tectonics, which was filled by marine/transitional sedimentary successions and is now occupied by wide flat coastal plains (Ascione et al., 2008). During the late Quaternary strong volcanic activity was registered in the coastal plain with the growth of the Somma-Vesuvius and the Campi Flegrei volcanoes (Romano et al., 1994). The landscape of the western portion of Campania is thus characterized by a wide flat area with isolated volcanic reliefs and islands. On the eastern side of the region the carbonate ridges transition to hilly landscapes of lower elevation, made up mainly of Miocene and Pliocene flysch successions. In this general orographic setting, SMCs are a widespread geomorphic unit along the flanks of the main carbonate ridges, as well as the slopes of the volcanoes, where they have higher longitudinal gradients. In the remaining hilly part of the region, stream catchments present lower mean longitudinal profiles, and wide alluvial plains linked to perennial river systems prevail.

The general climate is humid temperate with mean annual precipitation ranging from 1000 to $2000 \mathrm{~mm}$. In short, the main situations responsible for abundant rains over the region are generally northwesterly or westerly winds bringing eastward-moving cyclonic depressions. Due to the rugged topography of the region, heavy convective precipitation often results in flash floods, with concomitant widespread landslides.

The areas most affected by flash floods are the Lattari Mountains (Esposito et al., 2011), the Somma-Vesuvius area
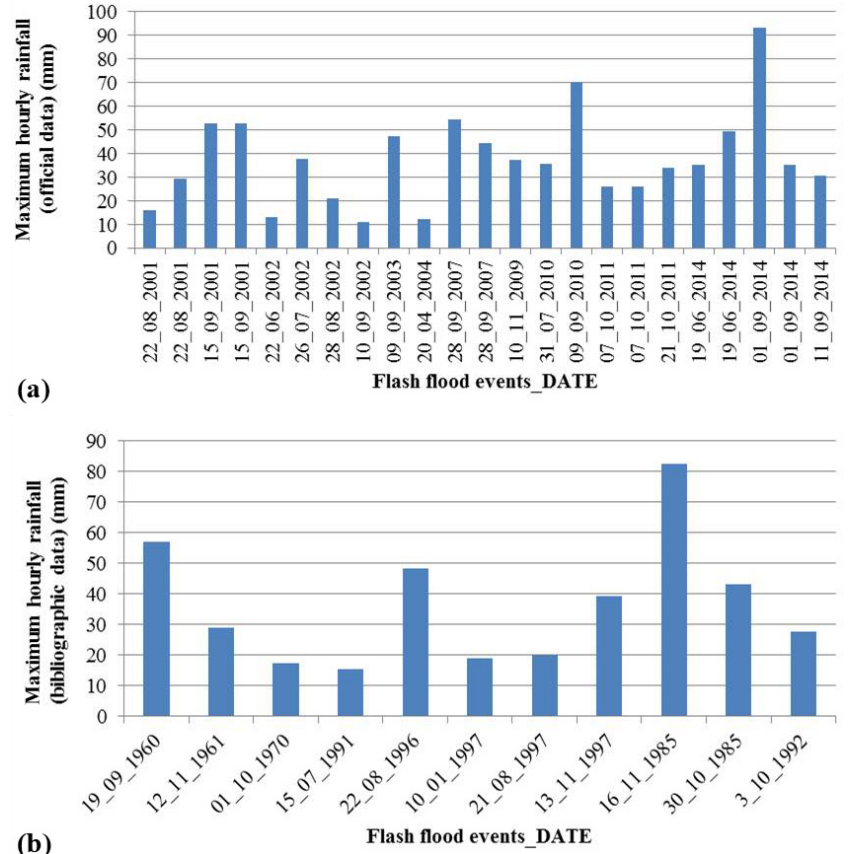

(b)

Flash flood events_DATE

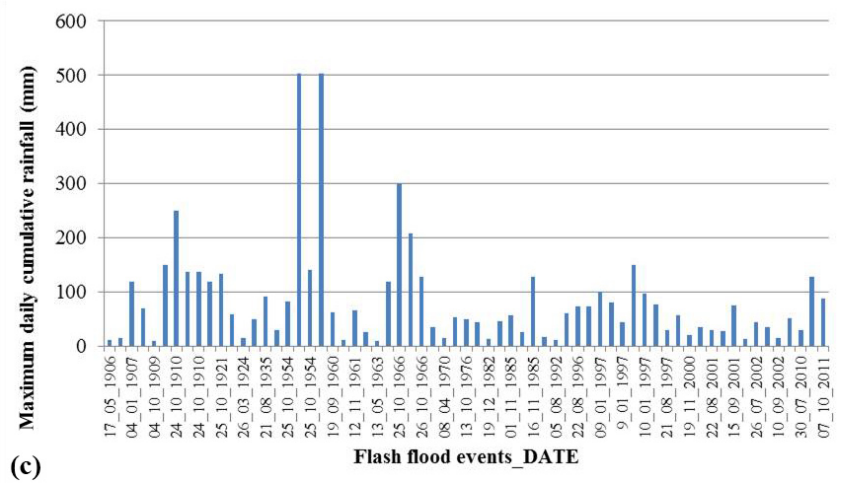

Figure 3. Rainfall data associated to flash floods: (a) maximum hourly rainfall (official data from CFDC); (b) maximum hourly rainfall (data from the literature); (c) maximum daily cumulative rainfall (data from SIMN).

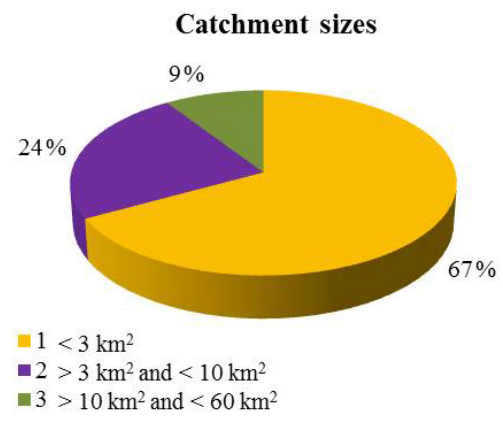

Figure 4. Distribution of flash flood events in the different catchment size classes: (1) $<3 \mathrm{~km}^{2}$; (2) $>3$ and $<10 \mathrm{~km}^{2}$; (3) $>10$ and $<60 \mathrm{~km}^{2}$. 


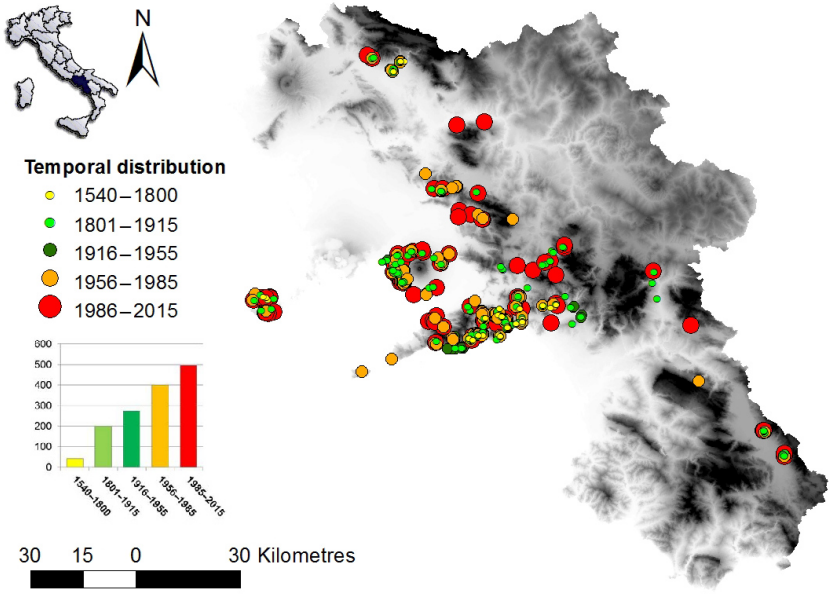

Figure 5. Temporal distribution of alluvial events in Campania. On the left a histogram illustrates their distribution over the time.

and the Island of Ischia (Santo et al., 2012; Alessio et al., 2013), the Picentini Mountains (Chirico et al., 2012; Santangelo et al., 2012; Santo et al., 2015), and the Matese, Caserta, and Maddalena mountain ridges (Santangelo et al., 2011, 2012; Scorpio et al., 2016). Given that the outlet zones of these torrential catchments are often highly urbanized, serious risk to population and settlements exists.

\section{Method}

\subsection{Data collection}

The main problem in searching for information about flash floods in Italy is that they are usually grouped with landslides and floods. In most cases the databases refer to landslides or floods or put different types of events together (landslides, flash floods in small catchments, floods in alluvial plains). As before stated, in Campania a regional-scale database on flash floods is not available.

For this reason, first of all we consulted the most complete archive of landslides and floods produced in Italy, the AVI project (an Italian acronym for Aree Vulnerate in Italia, areas affected by landslides or floods in Italy). It was commissioned by the Minister for Civil Protection to the National Group for the Prevention of Hydrogeologic Hazards of the Italian National Research Council (CNR) with a view to compile an inventory of information on areas historically affected by landslides and floods in the country (Guzzetti et al., 1994). We scrutinized the AVI archive to extract information about flash floods in small basins in the region. Since the archive is divided into landslides or floods, careful research was essential. Following this scrutiny, we also consulted and critically analyzed the existing literature related to flood events in Campania.

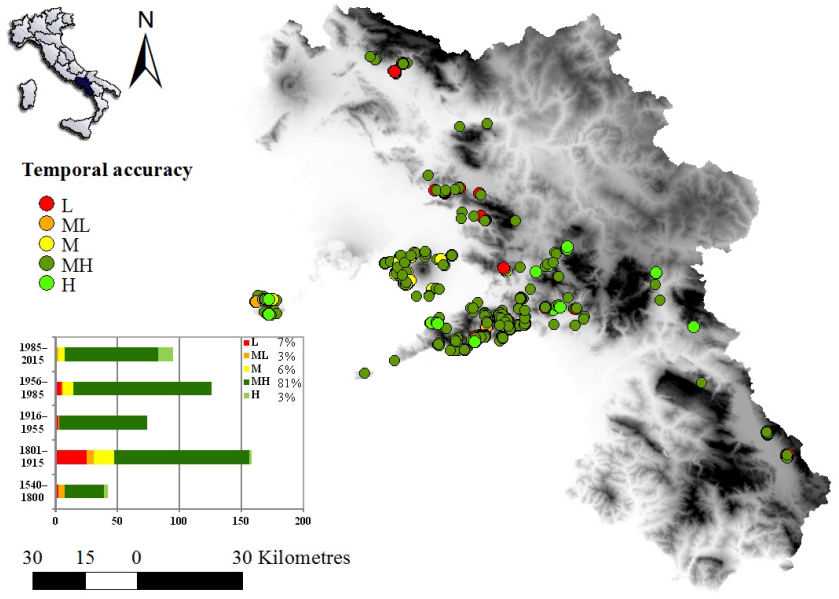

Figure 6. Temporal accuracy distribution of the events collected in Campania. The histogram shows the distribution in five classes of temporal accuracy: low (L), medium-low (ML), medium (M), medium-high $(\mathrm{MH})$, and high $(\mathrm{H})$. The histogram on the left shows the distribution of the accuracy classes in five temporal classes.

In order to discriminate flash floods from other hydrogeological events, we first adopted a "geographical" approach. Based on the evidence that all the recently occurring and well-documented flash flood events (i.e., with accurate event description and availability of rainfall data; Alessio et al., 2013; Chirico et al., 2012; Santangelo et al., 2011, 2012; Santo et al., 2002, 2012, 2015; Scorpio et al., 2016) affected SMCs, we catalogued all the events recorded in the outlet zone of this kind of hydrographic basin as "flash floods".

According to the above approach we distinguished the following four event types:

- floods in alluvial plains

- flash floods in SMCs

- landslides

- mixed and/or doubtful cases.

All the hydrogeological events that may be easily discriminated, based on general description and geographic location, were included in the database. For mixed and doubtful cases a second analysis was carried out, searching and consulting additional and new sources of information, in the attempt to classify them into one of the four types or to exclude them from the database. Once all the events were grouped into different types, we ruled out classes 1 and 3 for further study and focused on class 2, which represented $56 \%$ of the collected data (Fig. 2). We thus obtained a database of about 500 flash floods occurring in small catchments in Campania (Table 1). Unfortunately, information about damage and rainfall was not available in all cases. That said, the available chronicles and historical descriptions testify to sudden events, lasting just a few hours. Whenever possible, we tried to link the 


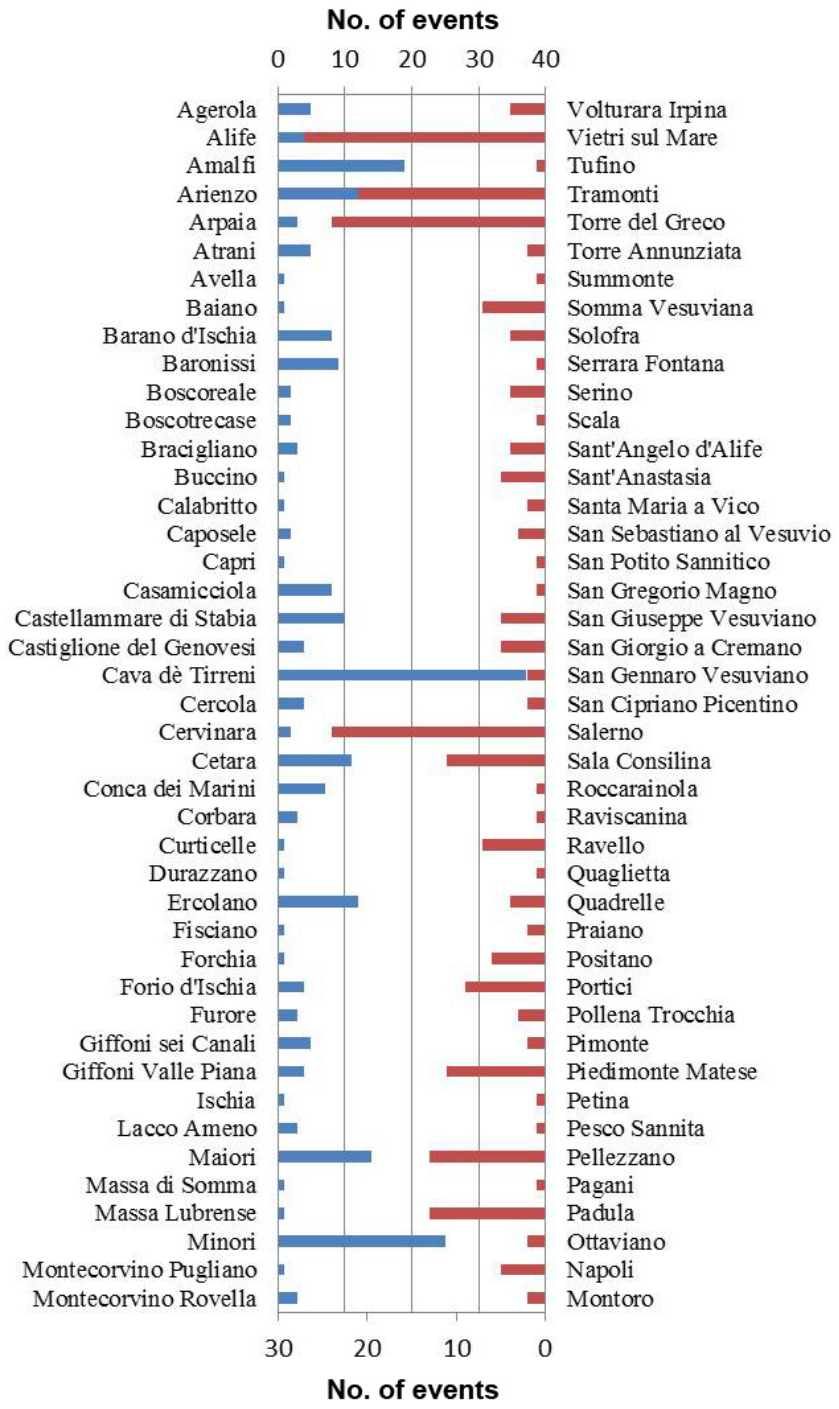

Figure 7. Recurrence of alluvial events in the municipalities.

documented events to hourly rainfall data obtained from the Campania Weather Forecasting Center (CFDC) (Fig. 3a) and rainfall data from the literature (Fig. 3b), obtaining a total record of 34 events. In all cases hourly rainfall values were very close to or greater than $30 \mathrm{~mm} \mathrm{~h}^{-1}$, and the daily rainfall obtained from the Italian Hydrologic and Oceanographic Service (SIMN) (Fig. 3c) was generally close to or greater than $100 \mathrm{~mm}$. These values are generally associated with high-intensity storms (Santo et al., 2012, 2015) and in the context of small catchments like those under study, characterized by very low concentration times (see Sect. 3.2), may be responsible for flash flood occurrence.

The repetitiveness of flash floods in the same area was also investigated. As shown in Fig. 7, among the 86 municipalities hit, 16 recorded more than 10 events. Nine out of these 16 municipalities were located at the outlet zone of coastal carbonate catchments (Sorrento Peninsula-Lattari ridge), whilst

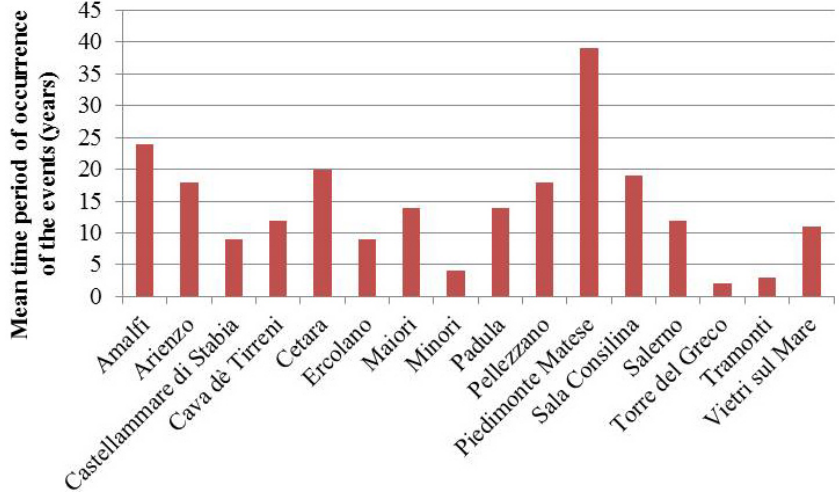

Figure 8. Mean time period of occurrence of the events in the municipalities that have recorded more than 10 events.

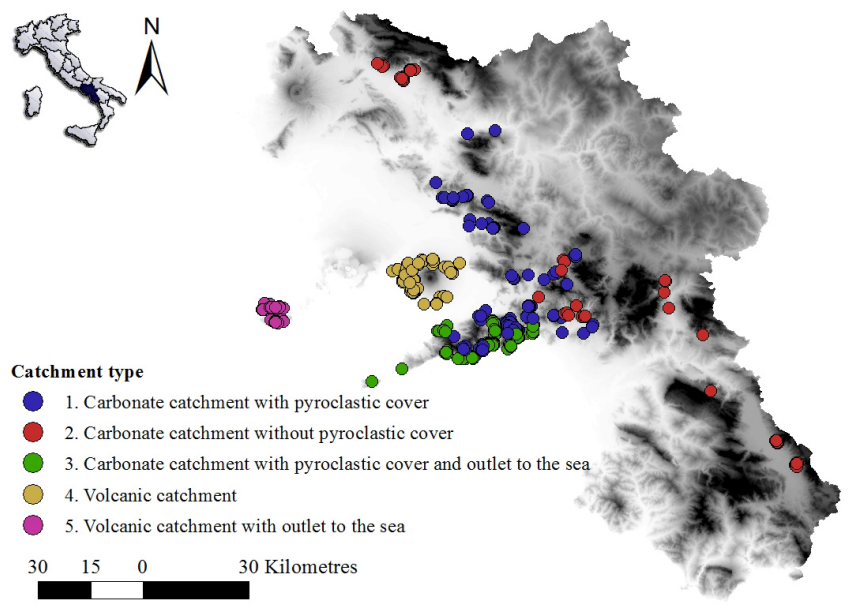

Figure 9. Geographical representation of the events, according to the catchment class.

the others are in the piedmont areas of inland carbonate massifs (Picentini and Matese mountains) or in the volcanic area of Mt. Somma-Vesuvius. In all these cases the mean time period of occurrence of the events is very low, ranging from 39 to 3 years, with a mean value of 15 (Fig. 8). If only the most damaging events are taken into account, the mean time period of occurrence increases to 50 years.

\subsection{Geomorphological features of the catchments involved}

The SMCs in Campania most severely affected by flash floods in recent decades are mainly located in carbonate and volcanic settings (Palma et al., 2009; Santangelo et al., 2012; Santo et al., 2012, 2015; Alessio et al., 2013). Regarding their geographical location, they may have both an inland outlet (generally represented by a fan or by a well-defined foothill area; Santangelo et al., 2012) or a coastal outlet, represented by a fan delta (Esposito et al., 2011; Santo et al., 2012). They also show similar morphometric conditions (Santangelo et 
Table 1. $N=$ ID event, date, municipality, location. CE means catchment classes: $(1)<3 \mathrm{~km}^{2} ;(2)>3$ and $<10 \mathrm{~km}^{2} ;(3)>10$ and $<60 \mathrm{~km}^{2}$. D means classes of damaged elements: (1) agricultural land; (2) architectural structures; (3) businesses; (4) private buildings; (5) roads; (6) underground utilities.

\begin{tabular}{|c|c|c|c|c|c|c|c|c|c|c|c|c|c|c|c|}
\hline $\mathrm{N}$ & Date & Municipality & Location & $\mathrm{CE}$ & $\mathrm{D}$ & $\mathrm{V}$ & $\mathrm{R}$ & $\mathrm{N}$ & Date & Municipality & Location & $\mathrm{CE}$ & $\mathrm{D}$ & $\mathrm{V}$ & $\mathrm{R}$ \\
\hline 1 & $08 / 10 / 1540$ & Amalfi & & 1 & & & 1 & 56 & $12 / 11 / 1817$ & Vietri sul Mare & Bonea catchment & 2 & & & 2 \\
\hline 2 & 1550 & Casamicciola & $\begin{array}{l}\text { La Pera quarry, } \\
\text { (Ervaniello) }\end{array}$ & 1 & 4 & & 11 & 57 & 1818 & Cava de' Tirreni & Amalfi coast & 1 & & & 2 \\
\hline 3 & $30 / 09 / 1581$ & Cava de' Tirreni & & 1 & & & 3 & 58 & 1819 & Cava de' Tirreni & Amalfi coast & 1 & & & 2 \\
\hline 4 & $30 / 09 / 1581$ & Salerno & & 1 & & & 3 & 59 & 1820 & Cava de' Tirreni & Amalfi coast & 1 & & & 2 \\
\hline 5 & $30 / 09 / 1581$ & Vietri sul Mare & & 2 & & & 3 & 60 & 1821 & Salerno & Irno catchment & 3 & & & 2 \\
\hline 6 & $01 / 10 / 1581$ & $\begin{array}{l}\text { Castiglione del Genovesi } \\
\text { De Petrinis land }\end{array}$ & & 1 & 4,3 & $700-1000^{*}$ & 8 & 61 & $06 / 06 / 1822$ & Sala Consilina & Monteoliveto square, & 2 & 4,3 & & 10 \\
\hline 7 & $01 / 10 / 1581$ & Giffoni sei Casali & & 2 & 4,3 & $700-1000^{*}$ & $3 ; 8$ & 62 & $27 / 10 / 1822$ & Corbara & & & & & 1 \\
\hline 8 & $01 / 10 / 1581$ & Piedimonte Matese & & 3 & 4,5 & 400 & 6 & 63 & $08 / 11 / 1822$ & Salerno & Road to Vietri & & & & 1 \\
\hline 9 & $01 / 10 / 1581$ & San Cipriano Picentino & & 1 & 4,3 & $700-1000^{*}$ & 8 & 64 & $24 / 01 / 1823$ & Amalfi & & 1 & & & 3 \\
\hline 10 & $31 / 08 / 1588$ & Atrani & & 2 & & & 3 & 65 & $24 / 01 / 1823$ & Bracigliano & & 1 & & & 3 \\
\hline 11 & 1626 & Salerno & Fusandola & 1 & 2 & & 1 & 66 & $24 / 01 / 1823$ & Cava de' Tirreni & & 1 & & & 3 \\
\hline 12 & $06 / 1643$ & Casamicciola & Bagni square & 1 & 4 & & 11 & 67 & $24 / 01 / 1823$ & Vietri sul Mare & & 2 & & & 3 \\
\hline 13 & $06 / 1643$ & Casamicciola & Piazza La Rita & 1 & 4 & & 11 & 68 & $08 / 10 / 1823$ & Corbara & Main square & 1 & & & 1 \\
\hline 14 & $20 / 12 / 1683$ & Maiori & Regina Major catchment & 3 & & & 1 & 69 & $03 / 10 / 1824$ & Minori & Loc. Torre & 2 & & & 1 \\
\hline 15 & $15 / 10 / 1696$ & Minori & Regina Minor catchment & 2 & & & $1 ; 2$ & 70 & 1825 & Cava de' Tirreni & Amalfi coast & 1 & & & 2 \\
\hline 16 & $26 / 09 / 1728$ & Piedimonte Matese & Vallata & 3 & 4,5 & & 6 & 71 & $18 / 06 / 1827$ & Sala Consilina & & 2 & 4,5 & & 6 \\
\hline 17 & $11 / 11 / 1773$ & Cava de' Tirreni & Passiano & 1 & 4 & $400^{*}$ & 1 & 72 & $11 / 07 / 1829$ & Arienzo & & 1 & 5 & & 6 \\
\hline 18 & $25 / 01 / 1736$ & Vietri sul Mare & Bonea catchment & 2 & & & 2 & 73 & $19 / 10 / 1830$ & Arienzo & & 1 & 5,1 & & 6 \\
\hline 19 & $26 / 09 / 1736$ & Vietri sul Mare & Bonea catchment & 2 & & & 2 & 74 & $16 / 07 / 1833$ & Arienzo & & 1 & & & 6 \\
\hline 20 & $11 / 1738$ & Vietri sul Mare & Bonea catchment & 2 & & & 2 & 75 & $13 / 09 / 1834$ & Cetara & Cetus catchment & 1 & & & 2 \\
\hline 21 & $03 / 11 / 1750$ & Salerno & Irno catchment & 3 & & & 3 & 76 & $18 / 07 / 1835$ & Cava de' Tirreni & Cavaiola catchment & 1 & & & 2 \\
\hline 22 & $03 / 11 / 1750$ & Vietri sul Mare & & 2 & & & 3 & 77 & $18 / 07 / 1835$ & Conca dei Marini & Irno and Bonea catchment & & & & 2 \\
\hline 23 & $10 / 10 / 1751$ & Amalfi & $\begin{array}{l}\text { Canneto catchment } \\
\text { (now Grevone) }\end{array}$ & 1 & & & 2 & 78 & $18 / 07 / 1835$ & Salerno & Irno catchment & 3 & & & 2 \\
\hline 24 & $01 / 09 / 1753$ & Amalfi & Loc. Chiorito & 1 & & & 1 & 79 & $27 / 09 / 1837$ & Salerno & Irno catchment & 3 & & & 2 \\
\hline 25 & $23 / 01 / 1757$ & Amalfi & $\begin{array}{l}\text { Canneto catchment } \\
\text { (now Grevone) }\end{array}$ & 1 & & & 2 & 80 & $27 / 09 / 1837$ & Vietri sul Mare & Bonea catchment & 2 & & & 2 \\
\hline 26 & $23 / 01 / 1757$ & Vietri sul Mare & Bonea catchment & 2 & & & 2 & 81 & $27 / 10 / 1839$ & Padula & & 2 & 5 & & 6 \\
\hline 27 & $09 / 10 / 1757$ & Amalfi & $\begin{array}{l}\text { Canneto catchment } \\
\text { (now Grevone) }\end{array}$ & 1 & & & 2 & 82 & $01 / 06 / 1841$ & Arienzo & & 1 & & & 6 \\
\hline 28 & $25 / 05 / 1762$ & Cetara & Cetus catchment & 1 & & & 3 & 83 & $20 / 09 / 1841$ & Piedimonte Matese & Loc. Vallata & 3 & 4,5 & $7^{*}$ & $6 ; 9$ \\
\hline 29 & $19 / 01 / 1764$ & Salerno & & & & & 2 & 84 & $20 / 09 / 1841$ & San Potito S. & & 1 & 4,5 & $7^{*}$ & $6 ; 9$ \\
\hline 30 & $11 / 1770$ & Salerno & Irno catchment & 3 & & & 2 & 85 & $07 / 11 / 1842$ & Serino & Loc. S.Rocco, S.Lucia & 1 & & 16 & 6 \\
\hline 31 & $11 / 11 / 1773$ & Cava de' Tirreni & & 1 & & & 3 & 86 & $26 / 10 / 1843$ & Cetara & Cetus catchment & 1 & & & 2 \\
\hline 32 & $11 / 11 / 1773$ & Cetara & & 1 & & & 3 & 87 & $26 / 10 / 1843$ & Maiori & Regina Major catchment & 3 & & & 2 \\
\hline 33 & $11 / 11 / 1773$ & Pellezzano & & 1 & & & 3 & 88 & $26 / 10 / 1843$ & Salerno & Irno catchment & 3 & & & 2 \\
\hline 34 & $11 / 11 / 1773$ & Salerno & Loc. Coperchia & 1 & 5 & 7 & $3 ; 1$ & 89 & $26 / 10 / 1843$ & Vietri sul Mare & Loc. Molina & 2 & & & 1 \\
\hline 35 & $11 / 11 / 1773$ & Tramonti & & 1 & & & 3 & 90 & $18 / 03 / 1845$ & Maiori & Regina Major catchment & 3 & & & 2 \\
\hline 36 & $11 / 11 / 1773$ & Vietri sul Mare & & 2 & & & 3 & 91 & $18 / 03 / 1845$ & Vietri sul Mare & Bonea catchment & 2 & & & 2 \\
\hline 37 & $20 / 11 / 1778$ & Alife & & 2 & 4 & & 6 & 92 & $01 / 10 / 1846$ & Amalfi & Canneto catchment(now Grevone) & 1 & & & 2 \\
\hline 38 & $20 / 11 / 1778$ & Piedimonte Matese & Loc.Vallata & 3 & 4 & & 9 & 93 & $01 / 10 / 1846$ & Baronissi & Irno catchment & 1 & & & 2 \\
\hline 39 & $02 / 1780$ & Atrani & Dragone catchment & 2 & & & 2 & 94 & $01 / 10 / 1846$ & Cetara & Cetus catchment & 1 & & & 2 \\
\hline 40 & $25 / 12 / 1796$ & Cava de' Tirreni & Cavaiola catchment & 1 & & & 2 & 95 & $01 / 10 / 1846$ & $\begin{array}{l}\text { Fisciano } \\
\text { and Irno catchments }\end{array}$ & Canneto, Regina Major, Bonea & 2 & & & 2 \\
\hline 41 & $25 / 12 / 1796$ & Salerno & Irno catchment & 3 & & & 2 & 96 & $01 / 10 / 1846$ & Maiori & Regina Major catchment & 3 & & & 2 \\
\hline 42 & $25 / 12 / 1796$ & Vietri sul Mare & Bonea catchment. & 2 & & & 2 & 97 & $01 / 10 / 1846$ & Pellezzano & Irno catchment & 1 & & & 2 \\
\hline 43 & $10 / 1803$ & Piedimonte Matese & Loc.Vallata & 3 & & & 6 & 98 & $31 / 12 / 1847$ & Amalfi & & 1 & & & 2 \\
\hline 44 & $21 / 01 / 1805$ & Solofra & Loc. Caposolofra & 2 & $5,4,2,3$ & & 15 & 99 & $13 / 09 / 1851$ & Alife & & 2 & & & 6 \\
\hline 45 & $22 / 01 / 1805$ & Serino & Ribottoli & 1 & & 67 & 6 & 100 & $13 / 09 / 1851$ & Piedimonte Matese & & 3 & & & 6 \\
\hline 46 & $09 / 06 / 1806$ & Sala Consilina & De Petrinisi street & 2 & 4,5 & 30 & 10 & 101 & $13 / 09 / 1851$ & Sant'Angelo d'Alife & & 2 & & & 6 \\
\hline 47 & $10 / 1810$ & Piedimonte Matese & Loc.Vallata & 3 & & & 9 & 102 & $21 / 11 / 1851$ & Serino & Loc. S.Lucia, Troiani & 1 & 4 & & 6 \\
\hline 48 & 1811 & Arienzo & & 1 & 5 & & 6 & 103 & 1851 & Padula & & 2 & 5 & & 6 \\
\hline 49 & 1811 & Santa Maria a Vico & & 1 & 5 & & 6 & 104 & 1851 & Volturara Irpina & & 2 & 5 & & 6 \\
\hline 50 & $21 / 12 / 1812$ & Positano & Parlati Mt. & 1 & 5 & 3 & 1 & 105 & $28 / 10 / 1852$ & Solofra & & 2 & $5,6,4$ & & 15 \\
\hline 51 & $30-31 / 07 / 1814$ & Bracigliano & & 1 & & & 1 & 106 & $05 / 01 / 1853$ & Vietri sul Mare & Bonea catchment. & 2 & & & 2 \\
\hline 52 & 04-19/12/1814 & Sala Consilina & $\begin{array}{l}\text { Vairo, Marroncelli and } \\
\text { Poerio street }\end{array}$ & 1 & 5 & & 10 & 107 & $20 / 03 / 1853$ & Volturara Irpina & & 2 & & & $6 ; 9$ \\
\hline 53 & 1814 & Piedimonte Matese & & 3 & & & 6 & 108 & $13 / 09 / 1857$ & Piedimonte Matese & Loc. Vallata & 3 & 4,5 & $90^{*}$ & $6 ; 9$ \\
\hline 54 & $12 / 11 / 1817$ & Cava de' Tirreni & Cavaiola catchment & 1 & & & 2 & 109 & $13 / 09 / 1857$ & Sant'Angelo d'Alife & Loc. S. Bartolomeo and S. Maria & 2 & 4,5 & $90^{*}$ & $6 ; 9$ \\
\hline 55 & $12 / 11 / 1817$ & Salerno & Irno catchment & 3 & & & 2 & 110 & 1857 & $\begin{array}{l}\text { Padula } \\
\end{array}$ & & 2 & 5 & & 6 \\
\hline
\end{tabular}


Table 1. Continued.

\begin{tabular}{|c|c|c|c|c|c|c|c|c|c|c|c|c|c|c|c|}
\hline 111 & $13 / 06 / 1858$ & Sala Consilina & $\begin{array}{l}\text { Indipendenza, Vairo, A. } \\
\text { Da Brescia, U. Bossi } \\
\text { and C. Battista streets }\end{array}$ & 2 & $4,1,5$ & 18 & $10 ; 6$ & 162 & $24 / 10 / 1910$ & Barano d'Ischia & Loc. Casabona & 1 & 4,5 & & $11 ; 2$ \\
\hline 112 & 1859 & Padula & & 2 & 4,5 & & 6 & 163 & 24-25/10/1910 & Boscotrecase & & 1 & & & $4 ; 2$ \\
\hline 113 & $08 / 1866$ & Maiori & $\begin{array}{l}\text { Loc. Cetraro, road to } \\
\text { Tramonti, Regina Major } \\
\text { catchment }\end{array}$ & 1 & & & 1,2 & 164 & $24 / 10 / 1910$ & Casamicciola & & 1 & 4,5 & 6 & $11 ; 2$ \\
\hline 114 & $11 / 11 / 1866$ & Vietri sul Mare & Bonea catchment & 2 & & & 2 & 165 & 24-25/10/1910 & Cercola & & 1 & & & $4 ; 2$ \\
\hline 115 & $16 / 03 / 1867$ & Vietri sul Mare & Bonea catchment & 2 & & & 2 & 166 & $24-25 / 10 / 1910$ & Ercolano & Loc. Resina & 1 & & & $4 ; 2$ \\
\hline 116 & $10 / 10 / 1867$ & Padula & & 2 & 4,5 & & 6 & 167 & $24 / 10 / 1910$ & Forio d'Ischia & Loc. Monterone & 1 & 4,5 & & $12 ; 2$ \\
\hline 117 & $01 / 04 / 1875$ & Conca dei Marini & & 1 & & & 2 & 168 & $24 / 10 / 1910$ & Furore & & 1 & 5 & & 3 \\
\hline 118 & 1876 & Padula & & 2 & 4,5 & & 6 & 169 & $24 / 10 / 1910$ & Ischia & & 1 & 5 & & $3 ; 2$ \\
\hline 119 & $01 / 02 / 1878$ & Conca dei Marini & & 1 & & & 2 & 170 & $24 / 10 / 1910$ & Lacco Ameno & & 1 & 4,5 & & $11 ; 2$ \\
\hline 120 & $01 / 02 / 1878$ & Salerno & Irno catchment & 3 & & & 2 & 171 & $24 / 10 / 1910$ & Maiori & $\begin{array}{l}\text { Loc. Erchie, S.Nicola } \\
\text { and Sovarano }\end{array}$ & 3 & 5,4 & 24 & $3 ; 15 ; 1$ \\
\hline 121 & $17 / 11 / 1880$ & Arienzo & & 1 & & & 9 & 172 & $24 / 10 / 1910$ & Minori & & 2 & 5 & 4 & $3 ; 1$ \\
\hline 122 & 1881 & Padula & & 2 & 4,5 & & 6 & 173 & $24-25 / 10 / 1910$ & Pollena Trocchia & & 1 & & & 4 \\
\hline 123 & 1883 & Padula & & 2 & 4,5 & & 6 & 174 & $24 / 10 / 1910$ & Portici & Giordano street & 1 & & & $4 ; 2$ \\
\hline 124 & $05 / 02 / 1885$ & Amalfi & $\begin{array}{l}\text { Canneto catchment } \\
\text { (now Grevone) }\end{array}$ & 1 & & & 2 & 175 & $24 / 10 / 1910$ & Ravello & & 1 & 6,5 & & $3 ; 1$ \\
\hline 125 & 1891 & Tramonti & Regina Major catchment & 1 & & & 2 & 176 & $24 / 10 / 1910$ & Salerno & Fusandola stream & 1 & 5 & & $3 ; 2$ \\
\hline 126 & 1896 & Baronissi & Irno catchment & 1 & & & 2 & 177 & $24-25 / 10 / 1910$ & San Sebastiano al Vesuvio & & 1 & & & $4 ; 2$ \\
\hline 127 & 1896 & Bracigliano & $\begin{array}{l}\text { Picentino, Fuorni and } \\
\text { Irno catchment }\end{array}$ & 1 & & & 2 & 178 & $24-25 / 10 / 1910$ & Sant'Anastasia & & 1 & & & 4 \\
\hline 128 & 1896 & Castiglione del Genovesi & Picentino catchment & 1 & & & 2 & 179 & $24 / 10 / 1910$ & Scala & Loc. Acquabona & 1 & 2 & & $3 ; 1$ \\
\hline 129 & 1896 & Conca dei Marini & $\begin{array}{l}\text { Picentino, Fuorni and } \\
\text { Irno catchment }\end{array}$ & 1 & & & 2 & 180 & $24 / 10 / 1910$ & Serrara Fontana & & 1 & 5 & & 11 \\
\hline 130 & 1896 & Salerno & Irno catchment & 3 & & & 2 & 181 & $24-25 / 10 / 1910$ & Somma Vesuviana & & 1 & & & 4 \\
\hline 131 & $07 / 10 / 1899$ & Calabritto & & 1 & $4,3,5$ & $100^{*}$ & 12 & 182 & $24 / 10 / 1910$ & Vietri sul Mare & loc. Molina & 2 & 5 & 1 & $3 ; 1 ; 2$ \\
\hline 132 & $07 / 10 / 1899$ & Caposele & & 1 & $4,3,5$ & $100^{*}$ & 12 & 183 & $02 / 01 / 1911$ & Cetara & Cetus catchment & 1 & & & 2 \\
\hline 133 & $07 / 10 / 1899$ & Castiglione del Genovesi & & 1 & $4,3,5$ & $100^{*}$ & 12 & 184 & $02 / 01 / 1911$ & Vietri sul Mare & Bonea catchment & 2 & & & 2 \\
\hline 134 & $07 / 10 / 1899$ & Cava de' Tirreni & & 1 & $4,3,5$ & $100^{*}$ & 12 & 185 & $21 / 09 / 1911$ & Boscoreale & & 2 & & & 4 \\
\hline 135 & $07 / 10 / 1899$ & Curticelle & & 1 & $4,3,5$ & $100^{*}$ & 12 & 186 & $21 / 09 / 1911$ & Ercolano & $\begin{array}{l}\text { Loc. Resina } \\
\text { (Pugliano, } \\
\text { Mare, Cortile, } \\
\text { Trentola streets) }\end{array}$ & 1 & & 6 & 4 \\
\hline 136 & $07 / 10 / 1899$ & Giffoni sei Casali & & 2 & $4,3,5$ & $100^{*}$ & 12 & 187 & $21 / 09 / 1911$ & Ottaviano & & 1 & & & 4 \\
\hline 137 & $07 / 10 / 1899$ & Giffoni Valle Piana & $\begin{array}{l}\text { Secco stream and } \\
\text { Colauro street }\end{array}$ & 3 & 4 & 3 & 12 & 188 & $21 / 09 / 1911$ & Portici & & 1 & & & 4 \\
\hline 138 & $07 / 10 / 1899$ & Montecorvino Pugliano & & 1 & $4,3,5$ & $100^{*}$ & 12 & 189 & $21 / 09 / 1911$ & San Giuseppe Vesuviano & & 1 & & & 5 \\
\hline 139 & $07 / 10 / 1899$ & Montecorvino Rovella & & 1 & $4,3,5$ & $100^{*}$ & 12 & 190 & $21 / 09 / 1911$ & Torre del Greco & $\begin{array}{l}\text { XX Settembre, } \\
\text { Nazionale, Fiorillo } \\
\text { and Umberto streets }\end{array}$ & 1 & & & 4 \\
\hline 140 & $07 / 10 / 1899$ & Quaglietta & & & $4,3,5$ & $100^{*}$ & 12 & 191 & $03 / 01 / 1915$ & Minori & $\begin{array}{l}\text { Regina Minor } \\
\text { catchment }\end{array}$ & 2 & & & 2 \\
\hline 141 & $07 / 10 / 1899$ & Salerno & $\begin{array}{l}\text { Irno catchment and } \\
\text { Rafastia torrent }\end{array}$ & 3 & $4,3,5$ & $100^{*}$ & 12,$5 ; 1$ & 192 & 1915 & Alife & & 2 & & & 6 \\
\hline 142 & $07 / 10 / 1899$ & Vietri sul Mare & Molina di Vietri & 2 & $4,3,5$ & 5 & 12,2 & 193 & $06 / 11 / 1916$ & Vietri sul Mare & Bonea catchment & 2 & & & 2 \\
\hline 143 & 1900 & Padula & & 2 & 4,5 & & 6 & 194 & $21 / 09 / 1921$ & San Giuseppe Vesuviano & & 1 & & & 4 \\
\hline 144 & $02 / 1903$ & Vietri sul Mare & Bonea catchment & 2 & & & 2 & 195 & $25 / 10 / 1921$ & Ercolano & & 1 & & & 4 \\
\hline 145 & 1903 & Cervinara & & 2 & 5 & & 1 & 196 & $25 / 10 / 1921$ & Portici & & 1 & & & 4 \\
\hline 146 & $07 / 10 / 1904$ & Ravello & Dragone catchment & 1 & & & 2 & 197 & $25 / 10 / 1921$ & San Giorgio a Cremano & & 1 & & & 4 \\
\hline 148 & $17-18 / 05 / 1906$ & Ercolano & & 1 & & 2 & 4 & 198 & $25 / 10 / 1921$ & Torre del Greco & & 1 & & & 4 \\
\hline 149 & $01 / 06 / 1906$ & Sant'Anastasia & & 1 & & & 4 & 199 & $13 / 11 / 1921$ & Furore & & 1 & & & 2 \\
\hline 150 & $01 / 06 / 1906$ & Cercola & & 1 & & & 4 & 200 & $26 / 03 / 1924$ & Agerola & & 1 & & $100^{*}$ & $3 ; 8$ \\
\hline 151 & $01 / 06 / 1906$ & Pollena Trocchia & & 1 & & & 4 & 201 & $26 / 03 / 1924$ & Amalfi & $\begin{array}{l}\text { Loc. Vettica Minore, } \\
\text { Baglio }\end{array}$ & 1 & $2,5,4$ & 60 & $3 ; 8$ \\
\hline 152 & 1906 & Torre del Greco & $\begin{array}{l}\text { Cavallerizzi, XX } \\
\text { Settembre and } \\
\text { Purgatorio streets, Del } \\
\text { Popolo square }\end{array}$ & 1 & & 26 & 4 & 202 & $26 / 03 / 1924$ & Atrani & & 2 & & $100^{*}$ & $3 ; 8$ \\
\hline 153 & $04 / 01 / 1907$ & Ercolano & & 1 & & & 4 & 203 & $26 / 03 / 1924$ & Cetara & & 1 & & $100^{*}$ & 1 \\
\hline 154 & $24-25 / 10 / 1908$ & Ercolano & & 1 & & 2 & 4 & 204 & $26 / 03 / 1924$ & Minori & & 2 & & $100^{*}$ & $3 ; 8$ \\
\hline 155 & $24-25 / 10 / 1908$ & Portici & & 1 & & & 4 & 205 & $26 / 03 / 1924$ & Positano & & 1 & & $100^{*}$ & $3 ; 8$ \\
\hline 156 & $24-25 / 10 / 1908$ & San Giorgio a Cremano & & 1 & & & 4 & 206 & $26 / 03 / 1924$ & Praiano & $\begin{array}{l}\text { Loc. Marina di } \\
\text { Praiano }\end{array}$ & 1 & 4 & 18 & $3 ; 8 ; 1 ; 2$ \\
\hline 157 & $24-25 / 10 / 1908$ & Torre del Greco & & 1 & & & 4 & 207 & $26 / 03 / 1924$ & Vietri sul Mare & $\begin{array}{l}\text { Bonea and Regina } \\
\text { Major catchments }\end{array}$ & 2 & 4 & $100^{*}$ & 5 \\
\hline 158 & 04/10/1909 & Boscotrecase & & 1 & & & 4 & 208 & $26 / 03 / 1924$ & Vietri sul Mare & & 2 & & & $3 ; 8$ \\
\hline 160 & $23 / 10 / 1910$ & Cetara & $\begin{array}{l}\text { Cetus catchment, Loc. } \\
\text { Utrio and Cappetta, } \\
\text { Federico street }\end{array}$ & 1 & 5,4 & $200^{*}$ & $15 ; 3 ; 1 ; 2 ; 13$ & 209 & $01 / 10 / 1927$ & Sala Consilina & & 2 & 4,5 & & 6 \\
\hline 161 & $24 / 10 / 1910$ & Amalfi & & 1 & 5 & 2 & $3 ; 1$ & 210 & $01 / 11 / 1927$ & Sala Consilina & Umberto I square & 2 & 4,5 & & 10 \\
\hline
\end{tabular}


Table 1. Continued.

\begin{tabular}{|c|c|c|c|c|c|c|c|c|c|c|c|c|c|c|}
\hline 211 & $21 / 09 / 1929$ & Giffoni Valle Piana & Picentino catchment & 3 & & 2 & 272 & $12 / 11 / 1961$ & Torre del Greco & & 1 & & 2 & 4 \\
\hline 212 & $21 / 09 / 1929$ & Montecorvino Rovella & & 1 & & 2 & 273 & $27 / 06 / 1962$ & San Giuseppe Vesuviano & & 1 & & & 5 \\
\hline 213 & $21 / 09 / 1929$ & Vietri sul Mare & Bonea catchment & 2 & & 2 & 274 & $16 / 02 / 1963$ & Cava de' Tirreni & Cavaiola catchment & 1 & & & 2 \\
\hline 214 & $31 / 08 / 1931$ & Castellammare di S. & & 1 & 1 & 1 & 275 & $16 / 02 / 1963$ & Pellezzano & Irno catchment & 1 & & & 2 \\
\hline 215 & $01 / 03 / 1935$ & Cava de' Tirreni & Cavaiola catchment & 1 & & 2 & 276 & $16 / 02 / 1963$ & Petina & & 1 & & & 2 \\
\hline 216 & $01 / 03 / 1935$ & Conca dei Marini & & 1 & & 2 & 277 & $16 / 02 / 1963$ & Positano & & 1 & & & 2 \\
\hline 217 & $01 / 03 / 1935$ & Minori & Regina Minor catchment & 2 & & 2 & 278 & $16 / 02 / 1963$ & Sala Consilina & & 2 & 4,5 & & 6 \\
\hline 218 & $01 / 03 / 1935$ & Ravello & Dragone catchment & 1 & & 2 & 279 & $16 / 02 / 1963$ & Tramonti & Regina Major catchment & 1 & & & 2 \\
\hline 219 & $01 / 03 / 1935$ & Tramonti & Regina Major catchment & 1 & & 2 & 280 & $18 / 02 / 1963$ & Padula & & 2 & 4,5 & & 6 \\
\hline 220 & $21 / 08 / 1935$ & Castellammare di S. & & 1 & & 1 & 281 & $21 / 02 / 1963$ & Positano & Loc. Trara Genoino & 1 & & & 1 \\
\hline 221 & 1935 & Giffoni Valle Piana & & 3 & & 1 & 282 & $13 / 05 / 1963$ & Sant'Anastasia & & 1 & & & 4 \\
\hline 222 & $14 / 09 / 1939$ & Amalfi & Canneto catchment (now Grevone) & 1 & & 2 & 283 & $30 / 05 / 1963$ & Torre del Greco & & 1 & & & 5 \\
\hline 223 & $14 / 09 / 1939$ & Conca dei Marini & Canneto & 1 & & 2 & 284 & $25 / 09 / 1963$ & Agerola & & 1 & & & 2 \\
\hline 224 & $14 / 09 / 1939$ & Maiori & Regina Major catchment & 3 & & 2 & 285 & $25 / 09 / 1963$ & Cava de' Tirreni & & 1 & & & 2 \\
\hline 225 & $01 / 06 / 1941$ & Arienzo & & 1 & & 9 & 286 & $25 / 09 / 1963$ & Cetara & Cetus catchment & 1 & & & 2 \\
\hline 226 & $18 / 06 / 1944$ & Minori & Regina Minor catchment & 2 & & 2 & 287 & $25 / 09 / 1963$ & Minori & & 2 & & & 2 \\
\hline 227 & $02 / 10 / 1945$ & Minori & Regina Minor catchment & 2 & & 2 & 288 & $25 / 09 / 1963$ & Pellezzano & Irno catchment & 1 & & & 2 \\
\hline 228 & $02 / 03 / 1947$ & Minori & Regina Minor catchment & 2 & & 2 & 289 & 07/10/1963 & Amalfi & Canneto catchment (now Grevone) & 1 & & & 2 \\
\hline 229 & $30 / 06 / 1947$ & Sala Consilina & Umberto I square & 24,5 & 1 & $9 ; 10$ & 290 & $07 / 10 / 1963$ & Cetara & Cetus catchment & 1 & & & 2 \\
\hline 230 & $25 / 10 / 1947$ & Minori & Regina Minor catchment & 2 & & 2 & 291 & $07 / 10 / 1963$ & Maiori & Regina Major catchment & 3 & & & 2 \\
\hline 231 & $23 / 05 / 1948$ & Minori & Regina Minor catchment & 2 & & 2 & 292 & $07 / 10 / 1963$ & Minori & Regina Minor catchment & 2 & & & 2 \\
\hline 232 & $26 / 07 / 1948$ & Somma Vesuviana & & 14 & & 4 & 293 & $07 / 10 / 1963$ & Salerno & & & & & 1 \\
\hline 233 & $05 / 09 / 1948$ & Minori & Regina Minor catchment & 2 & & 2 & 294 & $16 / 12 / 1963$ & Pellezzano & Irno catchment & 1 & & & 2 \\
\hline 234 & $02 / 10 / 1948$ & Alife & Loc. S.Michele & 25 & & 6 & 295 & $16 / 12 / 1963$ & Tramonti & Regina Major catchment & 1 & & & 2 \\
\hline 235 & $28 / 10 / 1948$ & Minori & Regina Minor catchment & 2 & & 2 & 296 & $13 / 01 / 1965$ & Torre del Greco & & 1 & & & 5 \\
\hline 236 & $01 / 10 / 1949$ & Vietri sul Mare & Bonea catchment & 2 & & 2 & 297 & $06 / 04 / 1966$ & Torre Annunziata & & 1 & & & 5 \\
\hline 237 & $14 / 08 / 1950$ & Somma Vesuviana & & 1 & & 4 & 298 & $25 / 10 / 1966$ & Castiglione del Genovesi & & 1 & & 1 & $3 ; 8$ \\
\hline 238 & $02 / 09 / 1950$ & Somma Vesuviana & & 1 & & 4 & 299 & $25 / 10 / 1966$ & Giffoni sei Casali & Monna Mt. & 2 & & 2 & $7 ; 1$ \\
\hline 239 & $25 / 12 / 1950$ & Castellammare di S. & Loc. Pozzano & 1 & & 1 & 300 & $26 / 10 / 1966$ & Alife & & 2 & & & 6 \\
\hline 240 & $21 / 01 / 1951$ & Minori & Regina Minor catchment & 2 & & 2 & 301 & $26 / 10 / 1966$ & Baronissi & & 1 & & & 3 \\
\hline 241 & $09 / 03 / 1951$ & Castellammare di S. & Loc. Pozzano & 1 & & 1 & 302 & $26 / 10 / 1966$ & Cava de' Tirreni & & 1 & & & 3 \\
\hline 242 & $09 / 11 / 1951$ & Giffoni Valle Piana & Picentino catchment & 3 & & 2 & 303 & $26 / 10 / 1966$ & Piedimonte Matese & Loc. Vallata & 3 & & & 6 \\
\hline 243 & $09 / 11 / 1951$ & Montecorvino Rovella & & 1 & & 2 & 304 & $26 / 10 / 1966$ & Salerno & & & & & 3 \\
\hline 244 & $11 / 09 / 1953$ & Agerola & & 1 & & 2 & 305 & 1966 & Ravello & & 1 & & & 1 \\
\hline 245 & $11 / 09 / 1953$ & Ravello & Dragone catchment & 1 & & 2 & 306 & $09 / 01 / 1968$ & Salerno & & & & & 5 \\
\hline 246 & 1953 & Ravello & Eastern side of Colonna Mt. & 1 & & 1 & 307 & $17 / 12 / 1968$ & Padula & & 2 & 4,5 & & 6 \\
\hline 247 & $25 / 10 / 1954$ & Amalfi & Canneto catchment (now Grevone) & 2 & & 3 & 308 & $19 / 12 / 1968$ & Amalfi & Canneto catchment (now Grevone) & 1 & & & 2 \\
\hline 248 & $25 / 10 / 1954$ & Atrani & & 2 & & 3 & 309 & $19 / 12 / 1968$ & Tramonti & Regina Major catchment & 1 & & & 2 \\
\hline 249 & $25 / 10 / 1954$ & Cava de' Tirreni & Loc. Alessia, Marini and Castagneto & 1 & 31 & $3 ; 1,18$ & 310 & 1968 & Alife & & 2 & & & 6 \\
\hline 250 & $25 / 10 / 1954$ & Maiori & & 3 & & $3 ; 1,18$ & 311 & $15 / 03 / 1969$ & Agerola & & 1 & & & 1 \\
\hline 251 & $25 / 10 / 1954$ & Minori & & 22 & & $3 ; 1,18$ & 312 & $15 / 03 / 1969$ & Cava de' Tirreni & Cavaiola catchment & 1 & & & 2 \\
\hline 252 & $25 / 10 / 1954$ & Positano & & 1 & & 3 & 313 & $17 / 09 / 1969$ & Cava de' Tirreni & Cavaiola catchment & 1 & & & 2 \\
\hline 253 & $25 / 10 / 1954$ & Praiano & Loc. Vettica Maggiore & 1 & & 3 & 314 & $22 / 09 / 1969$ & San Giorgio a Cremano & & 1 & & & 1 \\
\hline 254 & $25 / 10 / 1954$ & Salerno & Loc. Fratte, and Canalone & $36,4,5$ & $205^{*}$ & $3 ; 5 ; 18$ & 315 & 1969 & Arpaia & & 1 & & & 5 \\
\hline 255 & $25 / 10 / 1954$ & Tramonti & & 15 & & $3 ; 8 ; 2 ; 22$ & 316 & $08 / 04 / 1970$ & Salerno & & & 5 & 2 & 1 \\
\hline 256 & $25 / 10 / 1954$ & Vietri sul Mare & Loc. Di Molina and Marina & 2 & & 3,18 & 317 & $01 / 10 / 1970$ & Portici & & 1 & & 1 & 4 \\
\hline 257 & $05 / 11 / 1954$ & Ercolano & & 1 & & 4 & 318 & $01 / 10 / 1970$ & Torre Annunziata & & 1 & & 1 & 4 \\
\hline 258 & $04 / 02 / 1955$ & San Sebastiano al Vesuvio & & 1 & & 5 & 319 & $01 / 10 / 1970$ & Torre del Greco & & 1 & & & 4 \\
\hline 259 & $11 / 09 / 1955$ & Agerola & & 1 & & 2 & 320 & $02 / 10 / 1970$ & Amalfi & Canneto catchment (now Grevone) & 1 & & & 2 \\
\hline 260 & $11 / 09 / 1955$ & Pellezzano & Irno catchment & 1 & & 2 & 321 & $02 / 10 / 1970$ & Baronissi & Irno catchment & 1 & & & 2 \\
\hline 261 & $11 / 09 / 1955$ & Tramonti & Regina Major catchment & 1 & & 2 & 322 & $02 / 10 / 1970$ & Minori & & 2 & & & 2 \\
\hline 262 & $10 / 01 / 1956$ & San Giuseppe Vesuviano & & 1 & 3 & 4 & 323 & $02 / 10 / 1970$ & Pellezzano & Irno catchment & 1 & & & 2 \\
\hline 263 & $18 / 11 / 1956$ & Arpaia & & 1 & & 6 & 324 & $09 / 12 / 1970$ & Forio d'Ischia & Loc. Montevergine & 1 & & & 1 \\
\hline 264 & $21 / 01 / 1957$ & Sant'Anastasia & & 1 & & 5 & 325 & $25 / 12 / 1970$ & Amalfi & Canneto catchment (now Grevone) & 1 & & & 2 \\
\hline 265 & $22 / 10 / 1957$ & Cava de' Tirreni & & 1 & & 2 & 326 & $25 / 12 / 1970$ & Baronissi & Irno catchment & 1 & & & 2 \\
\hline 266 & $22 / 10 / 1957$ & Minori & Regina Minor catchment & 2 & & 2 & 327 & $25 / 12 / 1970$ & Minori & Regina Minor catchment & 2 & & & 2 \\
\hline 267 & $22 / 10 / 1957$ & Tramonti & Regina Major catchment & 1 & & 2 & 328 & $25 / 12 / 1970$ & Pellezzano & Irno catchment & 1 & & & 2 \\
\hline 268 & $30 / 12 / 1957$ & Cercola & & 1 & 2 & 4 & 329 & 1970 & Arienzo & & 1 & & & 6 \\
\hline 269 & $19 / 09 / 1960$ & Ercolano & & 1 & 1 & 4 & 330 & $19 / 01 / 1971$ & Torre del Greco & & 1 & & & 5 \\
\hline 270 & $19 / 09 / 1960$ & Portici & & 1 & & 4 & 331 & $21 / 02 / 1971$ & Castellammare di S. & & 1 & 5 & & 1 \\
\hline 271 & $07 / 07 / 1961$ & Torre del Greco & & 1 & 1 & 4 & 332 & $15 / 10 / 1971$ & Cava de' Tirreni & Cavaiola catchment & 1 & & & 2 \\
\hline
\end{tabular}

$\mathrm{V}=$ number of victims $\left({ }^{*}=\right.$ total number of victims per event on the same date); $\mathrm{R}=$ references (see Table 2$)$. 
Table 1. Continued.

\begin{tabular}{|c|c|c|c|c|c|c|c|c|c|c|c|c|c|c|c|}
\hline 333 & $15 / 10 / 1971$ & Tramonti & Regina Major catchment & 1 & & & 2 & 394 & $10 / 11 / 1987$ & Positano & & 1 & & & 2 \\
\hline 334 & $23 / 11 / 1971$ & Amalfi & $\begin{array}{l}\text { Canneto catchment } \\
\text { (now Grevone) }\end{array}$ & 1 & & & 2 & 395 & $10 / 11 / 1987$ & Ravello & & 1 & & & 2 \\
\hline 335 & 23/11/1971 & Minori & $\begin{array}{l}\text { Regina Minor } \\
\text { catchment }\end{array}$ & 2 & & & 2 & 396 & 10/11/1987 & Tramonti & Regina Major catchment & 1 & & & 2 \\
\hline 336 & $06 / 03 / 1972$ & Cava de' Tirreni & Cavaiola catchment & 1 & & & 2 & 397 & $13 / 11 / 1997$ & Ercolano & & & & & 4 \\
\hline 337 & $06 / 03 / 1972$ & Tramonti & Regina Major catchment & 1 & & & 2 & 398 & $15 / 09 / 1988$ & Baronissi & Irno catchment & 1 & & & 2 \\
\hline 338 & 27/07/1972 & Piedimonte Matese & Loc. Vallata & 3 & 4 & & 6 & 399 & $15 / 09 / 1988$ & Pellezzano & Irno catchment & 1 & & & 2 \\
\hline 339 & $21 / 10 / 1972$ & Cava de' Tirreni & Cavaiola catchment & 1 & & & 2 & 400 & $15 / 09 / 1988$ & Tramonti & Regina Major catchment & 1 & & & 2 \\
\hline 340 & $21 / 10 / 1972$ & Tramonti & Regina Major catchment & 1 & & & 2 & 401 & $15 / 07 / 1991$ & Torre del Greco & Santa Croce square & 1 & & & 4 \\
\hline 341 & $21 / 11 / 1972$ & Baronissi & Irno catch & 1 & & & 2 & 402 & $26 / 03 / 1992$ & Torre del Greco & port & 1 & & & 4 \\
\hline 342 & $21 / 11 / 1972$ & Cava de' Tirreni & Cavaiola catchment & 1 & & & 2 & 403 & $18 / 04 / 1992$ & Portici & & 1 & & & 4 \\
\hline 343 & $21 / 11 / 1972$ & Pellezzano & Irno catchment & 1 & & & 2 & 404 & $24 / 06 / 1992$ & Pellezzano & Loc.Cologna & 1 & & & 1 \\
\hline 344 & $02 / 01 / 1973$ & Amalfi & $\begin{array}{l}\text { Canneto catchment } \\
\text { (now Grevone) }\end{array}$ & 1 & & & 2 & 405 & $05 / 08 / 1992$ & Torre del Greco & & 1 & & & 4 \\
\hline 345 & $02 / 01 / 1973$ & Cava de' Tirreni & Cavaiola catchment & 1 & & & 2 & 406 & 25/09/1992 & Cava de' Tirreni & Cavaiola catchment & 1 & & & 2 \\
\hline 346 & $02 / 01 / 1973$ & Maiori & Regina Major catchment & 3 & & & 2 & 407 & $25 / 09 / 1992$ & Tramonti & Regina Major catchment & 1 & & & 2 \\
\hline 347 & $02 / 01 / 1973$ & Minori & $\begin{array}{l}\text { Regina Minor } \\
\text { catchment }\end{array}$ & 2 & & & 2 & 408 & 03-04/10/1992 & Torre del Greco & Piazza Palomba & 1 & & & 4 \\
\hline 348 & $02 / 01 / 1973$ & Tramonti & Regina Major catchment & 1 & & & 2 & 409 & 04/10/1992 & Baronissi & Irno catchment & 1 & & & 2 \\
\hline 349 & 19/09/1973 & Torre del Greco & & 1 & & 2 & 4 & 410 & 04/10/1992 & Cava de' Tirreni & Cavaiola catchment & 1 & & & 2 \\
\hline 350 & 1973 & Baiano & Loc. Lagno di Trulo & 1 & 5 & & 1 & 411 & $20 / 08 / 1993$ & Serino & Loc. Ribottoli, Puzzillo & 1 & & 1 & 6 \\
\hline 351 & $25 / 09 / 1974$ & Arienzo & & 1 & 4 & & 6 & 412 & 20/08/1993 & Solofra & Loc. Puzzillo & 2 & 3 & 1 & $18 ; 5 ; 1$ \\
\hline 352 & $25 / 09 / 1974$ & Arpaia & & 1 & 4 & & 6 & 413 & $08 / 12 / 1993$ & Cava de' Tirreni & Loc. Rotolo & 1 & & & 1 \\
\hline 353 & $25 / 09 / 1974$ & Forchia & & 1 & 4 & & 6 & 414 & $20 / 12 / 1993$ & Padula & & 2 & 4,5 & & 9 \\
\hline 354 & 03/10/1974 & Arienzo & & 1 & & & 6 & 415 & $26 / 12 / 1993$ & Sala Consilina & & 2 & 4,5 & & 6 \\
\hline 355 & $03 / 10 / 1974$ & Sant'Angelo d'Alife & & 2 & & & 6 & 416 & $22 / 08 / 1996$ & Massa di Somma & Paparo street & 1 & & & 4 \\
\hline 356 & $05 / 10 / 1974$ & Arienzo & & 1 & & & 6 & 417 & 22/08/1996 & San Gennaro Vesuviano & & 1 & & & 4 \\
\hline 357 & $28 / 06 / 1976$ & Salerno & Irno catchment & 3 & & & 1 & 418 & $22 / 08 / 1996$ & San Giorgio a Cremano & Matteotti street & 1 & & & 4 \\
\hline 358 & 13/10/1976 & Torre del Greco & & 1 & & 1 & 4 & 419 & 22/08/1996 & Torre del Greco & Port, XX Settembre street & 1 & & & 4 \\
\hline 359 & 29/10/1979 & Torre del Greco & Cavallo street & 1 & & 2 & 4 & 420 & 20/09/1996 & Cava de' Tirreni & Cavaiola catchment & 1 & & & 2 \\
\hline 360 & $12 / 10 / 1980$ & Cava de' Tirreni & Cavaiola catchment & 1 & & & 2 & 421 & $20 / 09 / 1996$ & Giffoni sei Casali & Picentino catchment & 3 & & & 2 \\
\hline 361 & $12 / 10 / 1980$ & Maiori & Regina Major catchment & 3 & & & 2 & 422 & 20/09/1996 & Tramonti & Regina Major catchment & 1 & & & 2 \\
\hline 362 & $12 / 10 / 1980$ & Minori & $\begin{array}{l}\text { Regina Minor } \\
\text { catchment }\end{array}$ & 2 & & & 2 & 423 & $26 / 11 / 1996$ & Padula & & 2 & 5 & & 6 \\
\hline 363 & $12 / 10 / 1980$ & Tramonti & Regina Major catchment & 1 & & & 2 & 424 & $26 / 11 / 1996$ & Sala Consilina & & 2 & 5 & & 6 \\
\hline 364 & $15 / 10 / 1980$ & Cava de' Tirreni & Cavaiola catchment & 1 & & & 2 & 425 & 09-10/01/1997 & Casamicciola & $\begin{array}{l}\text { loc. Montagnone, Molara, Cantoni, Tresta, } \\
\text { Cognola, Campomanno; La Pera, } \\
\text { Ervaniello and Puzzillo quarries, Mt. } \\
\text { Taburno, }\end{array}$ & 1 & & & 11 \\
\hline 365 & $01 / 06 / 1981$ & Forio d'Ischia & Paola Poli restaurant & 1 & 4 & & 5 & 426 & 09/01/1997 & Castellammare di S. & & 1 & & 4 & 5 \\
\hline 366 & 19/12/1982 & Torre del Greco & Cavallo street & 1 & & 2 & 4 & 427 & 09/01/1997 & Corbara & Chiunzi pass & 1 & & & 5 \\
\hline 367 & $15 / 08 / 1983$ & Barano d'Ischia & $\begin{array}{l}\text { Scura and Olmitello } \\
\text { quarries }\end{array}$ & 1 & & & 15 & 428 & 09-10/01/1997 & Lacco Ameno & Cito Mt. & 1 & & & 11 \\
\hline 368 & $30-31 / 10 / 1985$ & Ercolano & Palmieri street & 1 & & & 4 & 429 & 10/01/1997 & Castellammare di S. & Loc. Pozzano & 1 & 4,5 & & 14 \\
\hline 369 & $30-31 / 10 / 1985$ & Ottaviano & Cemetery & 1 & & & 4 & 430 & $10 / 01 / 1997$ & Cava de' Tirreni & Cinque street,SS18, Loc. Avvocatella & 1 & & & 1 \\
\hline 370 & $30-31 / 10 / 1985$ & Portici & Railway & 1 & & & 4 & 431 & $10 / 01 / 1997$ & San Cipriano Picentino & Loc. Campigliano & 1 & & & 1 \\
\hline 371 & $30-31 / 10 / 1985$ & San Gennaro Vesuviano & & 1 & & & 4 & 432 & $10 / 01 / 1997$ & San Giuseppe Vesuviano & & 1 & & & 4 \\
\hline 372 & $30-31 / 10 / 1985$ & Torre del Greco & Cavallo street & 1 & & & 4 & 433 & $10 / 01 / 1997$ & Vietri sul Mare & Loc. Guarno and Tresaro & 2 & & & 1 \\
\hline 373 & $02 / 11 / 1985$ & Ercolano & Palmieri street & & & 1 & 4 & 434 & $21 / 08 / 1997$ & Sant'Anastasia & & 1 & & & 4 \\
\hline 374 & $16-17 / 11 / 1985$ & Portici & & 1 & & & 4 & 435 & $21 / 08 / 1997$ & Somma Vesuviana & & 1 & & & 4 \\
\hline 375 & $16-17 / 11 / 1985$ & San Giorgio a Cremano & Tufarelli street & 1 & & & 4 & 436 & $13 / 11 / 1997$ & Boscoreale & Diaz street & 1 & & & 4 \\
\hline 376 & $16-17 / 11 / 1985$ & Torre del Greco & $\begin{array}{l}\text { Cavallo, Novesca, } \\
\text { Sant'Elena streets }\end{array}$ & 1 & & & 4 & 437 & 13/11/1997 & Cercola & & 1 & & & 4 \\
\hline 377 & $17 / 11 / 1985$ & Cava de' Tirreni & Cavaiola catchment & 1 & & & 2 & 438 & $13 / 11 / 1997$ & San Sebastiano al Vesuvio & & 1 & & & 4 \\
\hline 378 & $17 / 11 / 1985$ & Durazzano & Longano Mt. & 1 & & & 1 & 439 & 13/11/1997 & Somma Vesuviana & & 1 & & & 4 \\
\hline 379 & $17 / 11 / 1985$ & Maiori & Regina Major catchment & 3 & & & 2 & 440 & $13 / 11 / 1997$ & Torre del Greco & Beneduce street & 1 & & & 4 \\
\hline 380 & $17 / 11 / 1985$ & Summonte & & 1 & 6,5 & & 1 & 441 & 05/05/1998 & Avella & & 3 & & & 7 \\
\hline 381 & $17 / 11 / 1985$ & Tramonti & Regina Major catchment & 1 & & & 2 & 442 & 05/05/1998 & Montoro & & 1 & & 1 & 1 \\
\hline 382 & $01 / 02 / 1986$ & Castellammare di S. & Aragonese Castel & 1 & & & 5 & 443 & $24-25 / 7 / 1999$ & Casamicciola & Loc. Montagnone, Cantoni & 1 & 5 & & 11 \\
\hline 383 & $01 / 02 / 1986$ & Forio d'Ischia & & 1 & & & 5 & 444 & $24-25 / 7 / 1999$ & Lacco Ameno & Ervaniello quarry, La Rita & & 5 & & 15 \\
\hline 384 & $13 / 03 / 1986$ & Cava de' Tirreni & Loc. Molina & 1 & & & 2 & 445 & $15 / 12 / 1999$ & Cervinara & & 2 & & 5 & $5 ; 6$ \\
\hline 385 & 13/03/1986 & Pellezzano & Irno catchment & 1 & & & 2 & 446 & $19 / 11 / 2000$ & Torre del Greco & Loc. Santa Maria La Bruna & 1 & & & 4 \\
\hline 386 & 20/07/1986 & Roccarainola & & 1 & & & 6 & 447 & $27 / 12 / 2000$ & Ercolano & Caprile street & 1 & & & 4 \\
\hline 387 & $24 / 11 / 1986$ & Cava de' Tirreni & Cavaiola catchment & 1 & & & 2 & 448 & $22 / 08 / 2001$ & Santa Maria a Vico & & 1 & & & 6 \\
\hline 388 & $24 / 11 / 1986$ & Tramonti & Regina Major catchment & 1 & & & 2 & 449 & $22 / 08 / 2001$ & Sant'Angelo d'Alife & & 2 & $4,6,5$ & & 16 \\
\hline 389 & 22/02/1987 & Barano d'Ischia & & 1 & & & 6 & 450 & $15 / 09 / 2001$ & Barano d'Ischia & Olmitello quarry & 1 & 6 & & 15 \\
\hline 390 & $16 / 10 / 1987$ & Baronissi & Irno catchment & 1 & & & 2 & 451 & $15 / 09 / 2001$ & Casamicciola & Loc. La Rita & 1 & 4 & 2 & 11 \\
\hline 391 & $16 / 10 / 1987$ & Pellezzano & Irno catchment & 1 & & & 2 & 452 & $22 / 06 / 2002$ & Raviscanina & & 1 & 1 & & $9 ; 6$ \\
\hline 392 & $09 / 11 / 1987$ & Castellammare di S. & & 1 & & & 18 & 453 & $26 / 07 / 2002$ & Caposele & & 1 & 6,4 & & 18 \\
\hline 393 & $10 / 11 / 1987$ & Minori & $\begin{array}{l}\text { Regina Minor } \\
\text { catchment }\end{array}$ & 2 & & & 2 & 454 & $28 / 08 / 2002$ & Barano d'Ischia & Scura quarry & 1 & & & 15 \\
\hline
\end{tabular}


Table 1. Continued.

\begin{tabular}{|c|c|c|c|c|c|c|c|}
\hline 455 & $10 / 09 / 2002$ & Barano d'Ischia & Scura and Petrella quarries & 1 & & & 15 \\
\hline 456 & $23-24 / 09 / 2002$ & Barano d'Ischia & Olmitello quarry & 1 & 64 & & 15 \\
\hline 457 & $09 / 09 / 2003$ & Castellammare di S. & Castel & 1 & & & 18 \\
\hline 458 & $20 / 04 / 2004$ & Cava de' Tirreni & Loc. Badia and Sant'Arcangelo & 1 & 5,4 & & 18 \\
\hline 459 & $28 / 09 / 2007$ & Montoro & Loc. Frazione Chiusa and Aterrana & 1 & 5,4 & & 18 \\
\hline 460 & $28 / 09 / 2007$ & Volturara Irpina & Loc. Candragone & 2 & 4,3 & & $18 ; 6$ \\
\hline 461 & $10 / 11 / 2009$ & Casamicciola & Bagni square & 1 & 4,5 & 1 & 12 \\
\hline 462 & $30 / 07 / 2010$ & Somma Vesuviana & & 1 & & & 4 \\
\hline 463 & $31 / 07 / 2010$ & Giffoni sei Casali & Prepezzano stream, Loc. Madonna del Carmine & 3 & 5 & & 8 \\
\hline 464 & $09 / 09 / 2010$ & Atrani & & 2 & $5,4,1$ & 1 & 18 \\
\hline 465 & 07/10/2011 & Buccino & Loc. Teglia & 2 & 5,4 & & $17 ; 18$ \\
\hline 466 & $07 / 10 / 2011$ & San Gregorio Magno & Loc. Matruro, Teglia & 3 & 5 & & 18 \\
\hline 467 & $21 / 10 / 2011$ & Pollena Trocchia & & 1 & & 1 & 4 \\
\hline 468 & $19 / 06 / 2014$ & Arienzo & Pinazzola street & 1 & $5,4,3$ & & 18 \\
\hline 469 & $19 / 06 / 2014$ & Tufino & Loc. Icap, Vignola and Ferone, Turati street & 1 & 4 & & 18 \\
\hline 470 & $01 / 09 / 2014$ & Solofra & Loc. Madonna della Neve, Santa Lucia & 2 & $5,4$. & & 18 \\
\hline 471 & $01 / 09 / 2014$ & Volturara Irpina & Rimembranza street & 2 & 5,4 & & 18 \\
\hline 472 & $11 / 09 / 2014$ & Castellammare di S. & Loc. Quisisana & 1 & 5 & & 18 \\
\hline 473 & $25 / 02 / 2015$ & Barano d'Ischia & Loc. Olmitello & 1 & & 1 & 18 \\
\hline 474 & $19 / 09 / 1943$ & Quadrelle & Loc. Mugnano-Quadrelle & 2 & & & 18 \\
\hline 475 & $09 / 09 / 1973$ & Quadrelle & Loc. Mugnano-Quadrelle & 2 & & & 18 \\
\hline 476 & $20 / 08 / 1997$ & Quadrelle & Loc. Mugnano-Quadrelle & 2 & & & 18 \\
\hline 478 & 13/11/1997 & Quadrelle & Loc. Mugnano-Quadrelle & 2 & & & 18 \\
\hline
\end{tabular}

al., 2012; Alessio et al., 2013), which can be summed up as follows:

- limited catchment area (from a few $\mathrm{km}^{2}$ to $10 \mathrm{~km}^{2}$ )

- high relief energy (from hundreds of meters up to $1000 \mathrm{~m}$ )

- high slope gradient (generally greater than $35^{\circ}$ )

- high mean gradient of feeder channel (greater than $15^{\circ}$ )

- low concentration time (from 30 min to a few hours).

As the sediments captured by the stream and transported down valley generally come from the bed and banks of the torrent, we tried to discriminate among different catchment types, based on the following parameters:

- bedrock of the catchment (carbonate/volcanic);

- presence/absence of detrital cover and, if present, its origin (weathered bedrock, soil, pyroclastic cover, etc.);

- type of outlet zone (alluvial fan or fan delta in coastal area).

Thus the collected events were eventually grouped into the following five classes:
- carbonate catchment with pyroclastic cover

- carbonate catchment without pyroclastic cover

- carbonate catchment with pyroclastic cover and outlet to the sea

- volcanic catchment

- volcanic catchment with outlet to the sea.

\section{Results}

The database contains about 500 events (Table 1) and is being continually updated. Each event is identified by an ID and by its geographical coordinates (events are located with Google Earth ${ }^{\odot}$ ). In order to include an event in our database, knowledge of its location and its temporal occurrence was essential. The events were located as points, usually at the fan apex or where the main damage was recorded. The catchments were catalogued according to their size (column CE in Table 1) and, as shown in Fig. 4, 67\% of the events in question affected catchments smaller than $3 \mathrm{~km}^{2}$, while $91 \%$ of the catchments were smaller than $10 \mathrm{~km}^{2}$. Particular attention was given in the database to damage type (column D 


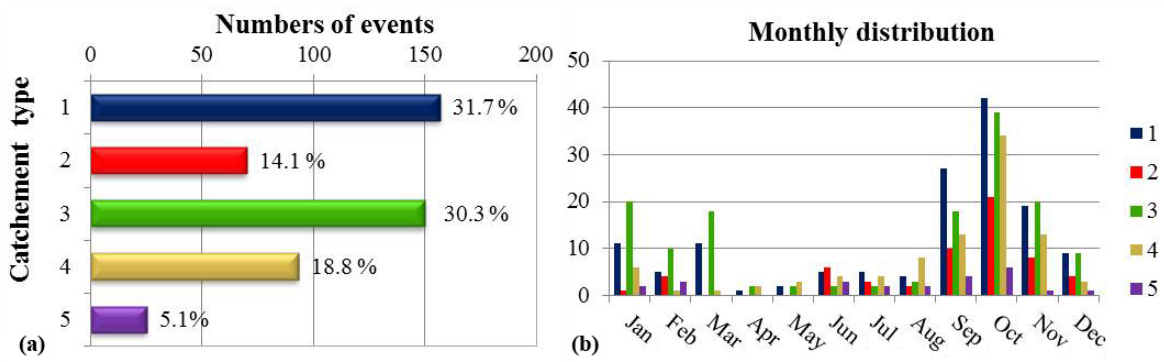

Figure 10. Number (a) and monthly distribution (b) of flash floods events for the different classes of catchments. Catchment class key: (1) carbonate catchment with pyroclastic cover; (2) carbonate catchment without pyroclastic cover; (3) carbonate catchment with pyroclastic cover and outlet to the sea; (4) volcanic catchment; (5) volcanic catchment with outlet to the sea.

Table 2. References used in Table 1 (R column).

\begin{tabular}{ll}
\hline References & No. \\
\hline Migale and Milone (1998) & 1 \\
Porfido et al. (2013) & 2 \\
Esposito et al. (2011) & 3 \\
Alessio et al. (2013) & 4 \\
Vallario (2001) & 5 \\
Santangelo et al. (2012) & 6 \\
Di Crescenzo and Santo (2005) & 7 \\
Esposito and Galli (2011) & 8 \\
Scorpio (2011) & 9 \\
Santangelo et al. (2011) & 10 \\
Santo et al. (2012) & 11 \\
Esposito et al. (2011) & 12 \\
ISPRA-Servizio Geologico d'Italia (2006) & 13 \\
Calcaterra and Santo (2004) & 14 \\
Del Prete and Mele (2006) & 15 \\
Di Crescenzo et al. (2013) & 16 \\
Chirico et al. (2012) & 17 \\
Newspapers & 18 \\
\hline
\end{tabular}

in Table 1) and to the presence of victims, if reported (column V in Table 1). The documented events caused serious social damage, primarily involving buildings, infrastructures, and lifelines (roads, pipelines, etc.). Damage to the population includes the number of victims, injured, homeless, and missing people. In several cases the information source does not allow casualties to be quantified with precision, since the information reported is typically generic, such as "some victims" or "several victims". Thus, in order not to loose any useful data, information about damage is also reported as text, as in the sources. Further, additional useful information can be included in the field notes.

The information thereby gathered may be useful to depict a regional scenario on the spatial and temporal distribution of flash floods in Campania. The oldest event occurred in 1540 in the town of Amalfi. The temporal distribution of the events in the region (Fig. 5) reveals that most of the municipalities have been affected by flash floods more than once during the time period covered by the database. Although there are several catchments with similar geological and geomorphological characteristics, many villages have no event recorded, probably related to the absence of inhabited areas. More than $60 \%$ of the events have occurred during the last 50 years, a finding which is probably related to the greater availability of information sources in recent times, the numerous scientific studies carried out in the last few decades and the growing attention to geological hazards. Five degrees of temporal accuracy were used to classify the different levels of knowledge for time occurrence of the events. Events for which only the year of occurrence is known have a low accuracy, while a high accuracy is assigned when hour, day, month, and year of occurrence are known. The histogram in Fig. 6 indicates that most of the events in question have a medium-to-high accuracy, meaning that day, month, and year of occurrence are known. The accuracy degree declines, as expected, for the oldest events.

Regarding the geomorphological features of the catchments involved, Fig. 9 shows the regional distribution of events, classified according to the five classes mentioned above. Most of the events took place in carbonate catchments with pyroclastic cover, both with and without an outlet to the sea (Figs. 9 and 10a). The widespread presence of carbonate catchments, as well as the high urbanization, affected the information source: as mentioned above, intense urbanization typically means greater availability of data, due to higher attention towards the damage generated by natural hazards. Distinguishing between different catchment types is important in order to discern the different bed and bank materials available which the flow could entrap and transport down valley. In a typical carbonate catchment the surge could generally take gravels without or with a low content of matrix (Fig. 11a); in carbonate catchments with pyroclastic cover, however, medium and coarse gravels with a high percentage of matrix are expected to be available (Fig. 11b). Further, in pure volcanic catchments, due to greater erodibility of the material, mainly silts and clays can be found (Fig. 11c). The mean time period of occurrence of the events in each catchment type ranges from 3 to 35 years. Carbonate catchments 


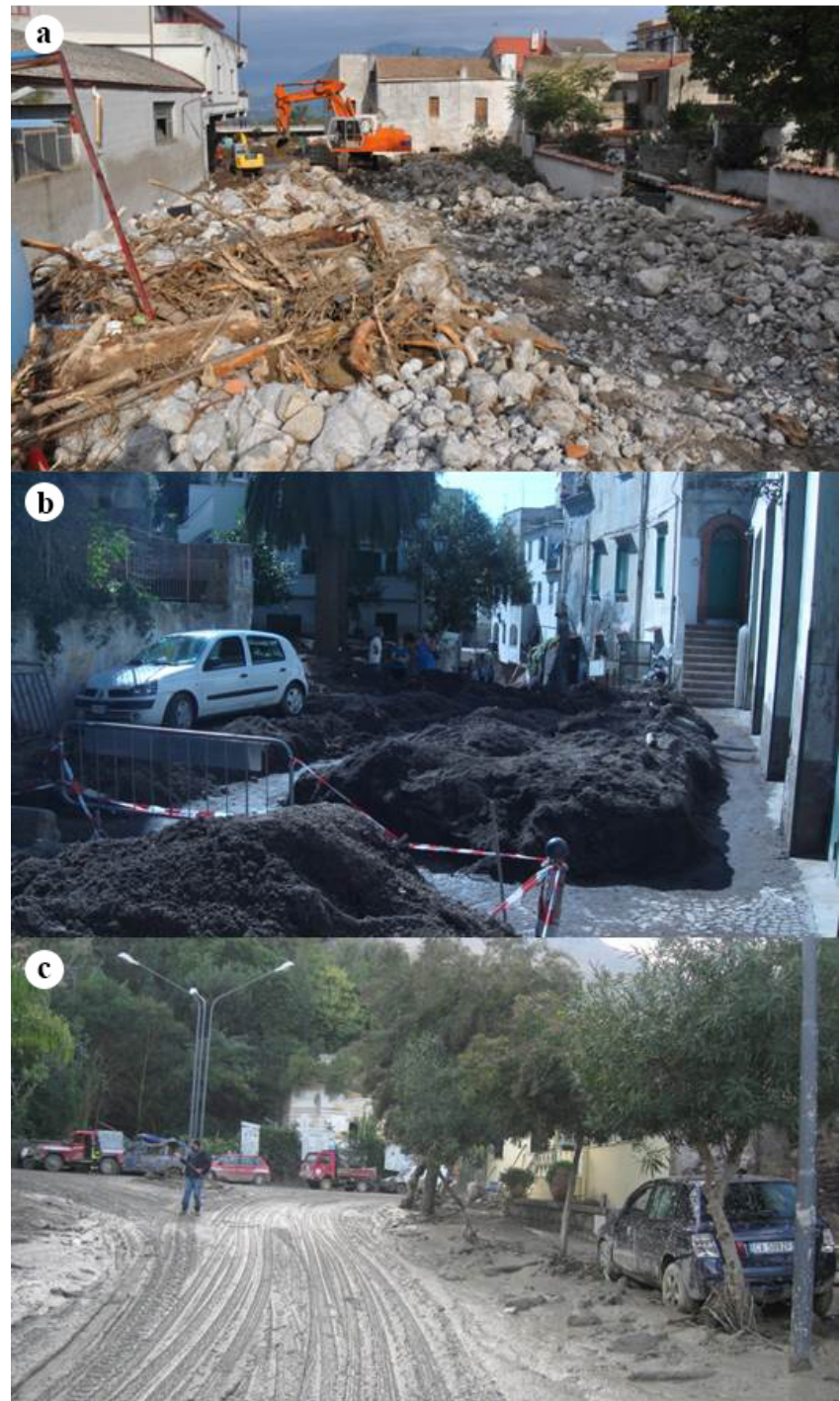

Figure 11. Different grain size deposits for the different catchment classes: (a) Solopaca 2015, carbonate gravels and blocks (class 2); (b) Atrani 2010, gravel and sands (classes 1 and 3); (c) Casamicciola 2009: sands, silt and clay (classes 4 and 5).

with pyroclastic cover, both with and without an outlet to the sea, show a mean time period of occurrence of 6 years, which becomes 10 years for carbonate catchments without pyroclastic cover. Regarding the volcanic catchments, which in general present a lower number of events, the mean time period of occurrence is 35 years at Ischia and 3 years for the area surrounding Mt. Somma-Vesuvius.

The monthly distribution of flash flood events is quite variable in the different types of catchments. Figure 10b shows that all the events peak in October, with autumn being the season with the highest frequency. After the dry period, heavy rainfall can generate sudden high discharge, and runoff can carry downstream sediments accumulating as a result of the erosion processes. Locally, this could also be related to

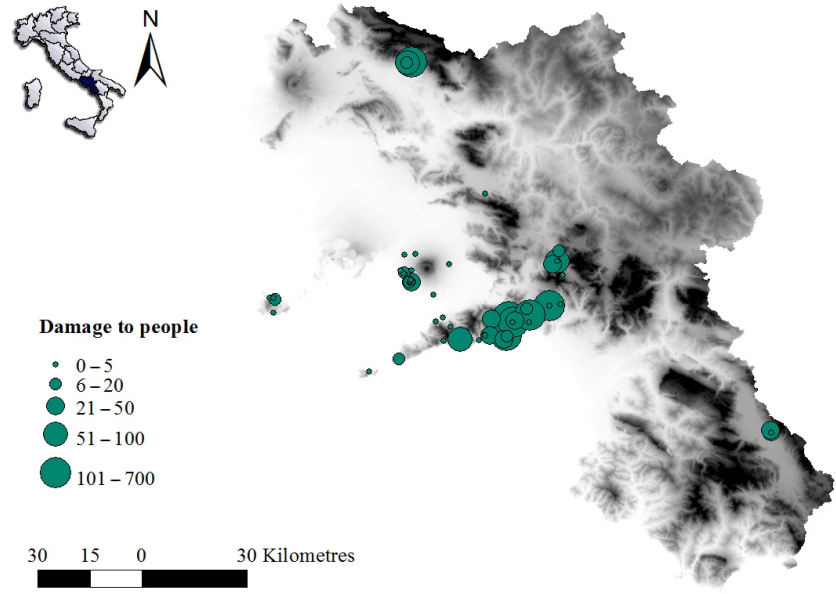

Figure 12. Distribution of victims recorded in the territory.

occurrence of wildfires during the dry season (Calcaterra et al., 2007a, b).

On the basis of the collected data, in the carbonate catchments with pyroclastic cover the lowest number of events is recorded in April, or generally during the spring, if there is an outlet to the sea. In the carbonate catchments without cover during spring (March-May) there are no events, and the lowest number of alluvial events is in January. In volcanic catchments, February and March are the months with the smallest number of events. For catchments with an outlet to the sea, during the spring (March-May) no event was recorded, and November-December are the months with the fewest events.

Regarding damage to people, in most cases the reported information is very generic, or in other cases the source provides the total number of victims per event but not for the individual municipalities. A precise estimate of the number of victims is thus not easy. In some cases divergences exist in the numbers of casualties reported by different sources for the same event. The differences could be due to several reasons, including the fact that the exact number is typically available only at the end of the search and rescue operations, from a few days to weeks after the event. During this period, newspapers and even official reports may provide different and changing data (Salvati et al., 2010). Typically, information sources mainly document the most severe events in terms of the number of deaths and damage caused.

About $18 \%$ of the events in the database caused at least one victim. Figure 12 depicts their distribution in the region. The most dangerous events hit the province of Salerno, affecting the carbonate catchments with pyroclastic cover, both with and without an outlet to the sea. This means that carbonate catchments with pyroclastic cover (class 1) are the most hazardous. All the events with more than 100 victims took place in October; further, for the most damaging events the total number of deaths was also caused by landslides. Hence it is not easy to evaluate the victims caused only by flash 


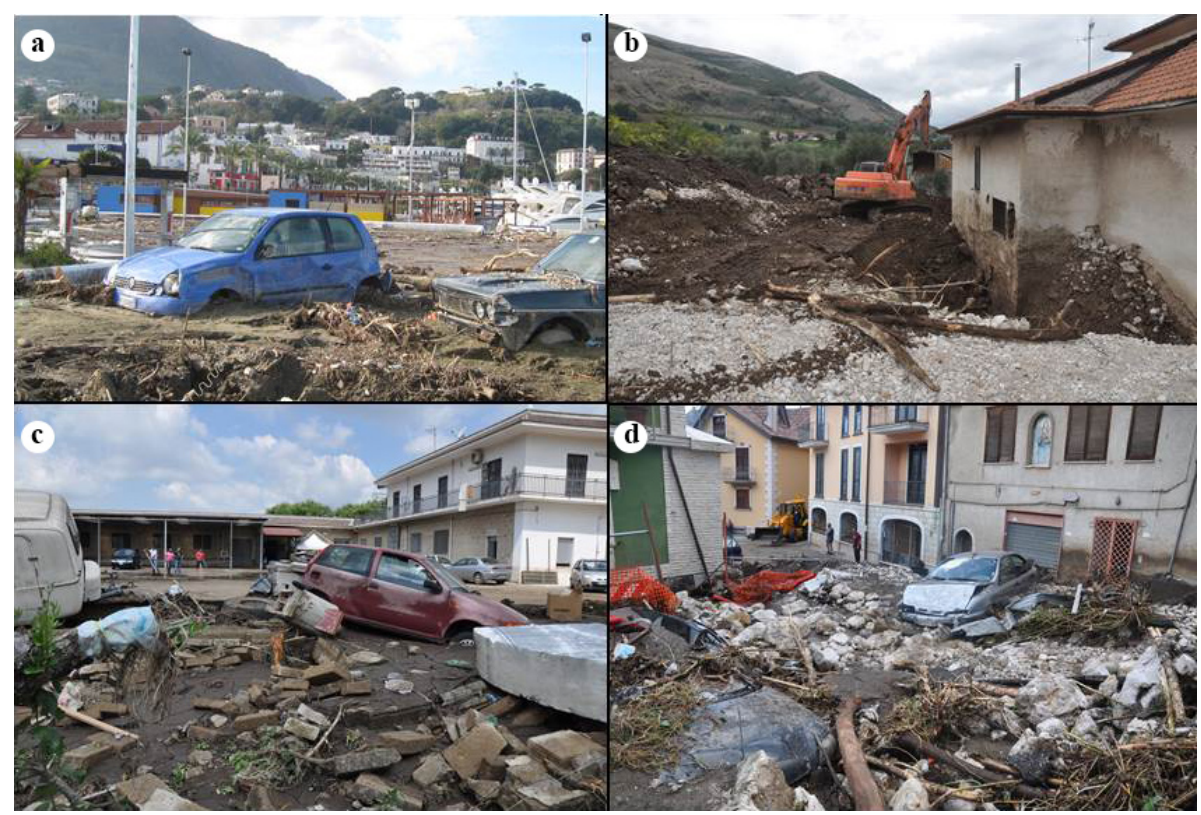

Figure 13. Typical examples of damage caused by alluvial events: (a) Casamicciola, 10 November 2009; (b) Buccino-Teglia, 7 October 2011; (c) Arienzo, 6 June 2014; (d) Solofra, 1 September 2014.

flood events in small catchments. Further, attribution of the number of casualties to each municipality was very difficult, since the source typically provides the total number of victims per event, not distinguishing the different villages. This applies, for instance, to the events in 1581 (700-1000 victims), in 1899 (approximately 100 victims), in 1924 (approximately 100 victims), and in 1910 (over 200 victims) which were the most devastating in recorded history.

The mean time period of occurrence for the events with more or less than 100 victims is very different: 62 years for the events with more than 100 victims and only 3 years for all the other events.

Taking into account the total number of events, the database contains few cases with injured, homeless and missing people. With regard to the homeless, for instance, the number is too small when compared with the numbers of events that caused complete destruction of the buildings. This suggests that the documented data in some ways underestimate reality.

The flash floods documented caused severe social damage, primarily involving buildings, lifelines and infrastructures. In particular, roads and private buildings were the most severely affected categories. In this regard, the most dangerous catchment types are carbonate catchments with and without pyroclastic cover. The damaged elements by each event are reported in classes in the database. In Fig. 13 some examples of damage produced by flash floods are shown.

\section{Final remarks}

Through an analysis of the existing literature, we focused on flash flood events in Campania, a region that has repeatedly been affected by severe flooding causing serious damage and fatalities. We collected temporal and spatial information on about 500 flash floods, thus building the first specific database concerning this type of geological hazard in the region. For this purpose, a critical scrutiny of the existing literature was performed to provide a degree of reliability for the collected information. We also defined the accuracy related to the temporal information available, and for most of the events accuracy proved to be medium to high, meaning that day, month and year of occurrence of the single event are known.

In order to reconstruct flood scenarios, we classified different catchment types on the basis of the main geological (bedrock lithology and presence/absence of detrital cover) and geomorphological parameters (type of outlet zone). This differentiation may be useful to understand the type of transported bed load (coarse vs. fine-grained) and to characterize the deposition area.

We also collected information about damage to people and society, which occurred as a consequence of flash floods. Most of the events took place in carbonate catchments with pyroclastic cover, both with and without an outlet to the sea. Among the 86 municipalities that were damaged by torrential flooding, 16 recorded more than 10 events. In Campania the mean occurrence interval of floods is very low, ranging from a few years for damage to buildings and infrastructures up to some decades in the case of events with victims. The 
widespread presence of carbonate catchments in the region and the intense urbanization of the area affected the information sources, and these represent the main weaknesses of the historical documents. Although the volcanic areas in Campania are densely populated, the alluvial events in carbonate catchments with pyroclastic cover caused more damage to people and society than those occurring in volcanic catchments. The study also showed that a significant number of catchments were affected by floods with high mean time period of occurrence of the events. The loss of historical memories of these events certainly lies at the origin of an increase in the risk conditions.

Such a database may be useful in different ways for land use and civil protection planning. First of all, it may help to locate the areas more susceptible to flash floods all over the region. Secondly, it may facilitate local authorities in charge of land management to select sites where monitoring procedures and/or prevention and mitigation works need to be adopted. In this perspective the main users of the database will be local administrators and the civil protection agency.

\section{Data availability}

The database was built for the author's PhD project; we have chosen to make it public with this publication. Any additional information about data sets can be provided by the authors.

Acknowledgements. We acknowledge the anonymous reviewers for their constructive comments, that have helped us to improve the manuscript.

Edited by: K. Schröter

Reviewed by: five anonymous referees

\section{References}

Alessio, G., De Falco, M., Di Crescenzo, G., Nappi, R., and Santo, A.: Flood hazard of the Somma-Vesuvius region based on historical (19-20th century) and geomorphological data, Ann. Geophys.-Italy, 56, S0434, doi:10.4401/ag-6440, 2013.

Antronico, L., Allasia, P., Baldo, M., Greco, R., Robustelli, G., and Sorriso-Valvo, M.: The use of airborne LiDAR data in basin-fan system monitoring: An example from southern Calabria (Italy), in: Eng. Geol. for Society and Territory - Volume 2: Landslide Processes, 441-444, 2015a.

Antronico, L., Greco, R., and Sorriso-Valvo, M.: Recent alluvial fans in Calabria (southern Italy), J. Maps, 3, 1-12, doi:10.1080/17445647.2015.1047905, 2015b.

Arattano, M., Marchi, L., and Cavalli, M.: Analysis of debrisflow recordings in an instrumented basin: confirmations and new findings, Nat. Hazards Earth Syst. Sci., 12, 679-686, doi:10.5194/nhess-12-679-2012, 2012.

Arattano, M., Cavalli, M., Comiti, F., Coviello, V., Macconi, P., and Marchi, L.: Standardization of methods and procedures for debris flow seismic monitoring, in: Eng. Geol. for Society and Territory - Volume 3, Springer International Publishing, 63-67, 2015.

Ascione, A., Cinque, A., Miccadei, E., Villani, F., and Berti, C.: The Plio-Quaternary uplift of the Apennine chain: new data from the analysis of topography and river valleys in Central Italy, Geomorphology, 102, 105-118, 2008.

Berti, M. and Simoni, A.: DFLOWZ: A free program to evaluate the area potentially inundated by a debris flow, Comput. Geosci., 67, 14-23, 2014.

Blahut, J., Glade, T., and Sterlacchini, S.: Debris flows risk analysis and direct loss estimation: the case study of Valtellina di Tirano, Italy, J. Mt. Sci., 11, 288-307, 2014.

Borga, M., Boscolo, P., Zanon, F., and Sangati, M.: Hydrometeorological analysis of the 29 August 2003 flash flood in the Eastern Italian Alps, J. Hydrometeorol., 8, 1049-1067, 2007.

Calcaterra, D. and Santo, A.: The January 10, 1997 Pozzano landslide, Sorrento Peninsula, Italy, Eng. Geol., 75, 181-200, 2004.

Calcaterra, D., Parise, M., Palma, B., and Pelella, L.: Multiple debris flows in volcaniclastic materials mantling carbonate slopes, in: Proceedings 2nd International Conference on "Debris-Flow Hazards Mitigation: Mechanics, Prediction, and Assessment", Taiwan, edited by: Wieczorek, G. F. and Naeser, N. D., 99-107, 2000.

Calcaterra, D., Parise, M., and Palma, B.: Combining historical and geological data for the assessment of the landslide hazard: a case study from Campania, Italy, Nat. Hazards Earth Syst. Sci., 3, 316, doi:10.5194/nhess-3-3-2003, 2003.

Calcaterra, D., Coppin, D., de Vita, S., Di Vito, M. A., Orsi, G., Palma, B., and Parise, M.: Slope processes in weathered volcaniclastic deposits within the city of Naples: the Camaldoli Hill case, Geomorphology, 87, 132-157, 2007a.

Calcaterra, D., Parise, M., Strumia, S., and Mazzella, E.: Relations between fire, vegetation and landslides in the heavily populated metropolitan area of Naples, Italy, edited by: Schaefer, V. R., Schuster, R. L., and Turner, A. K., in: Proceedings 1st North American Landslide Conference, Vail (Colorado), 3-8 June 2007, AEG Special Publ., 23, 1448-1461, 2007 b.

Carrara, A., Crosta, G., and Frattini, P.: Comparing models of debris-flow susceptibility in the alpine environment, Geomorphology, 94, 353-378, 2008.

Cascini, L., Cuomo, S., and Guida, D.: Typical source areas of May 1998 flow-like mass movements in the Campania region, Southern Italy, Eng. Geol., 96, 107-125, 2008a.

Cascini, L., Ferlisi, S., and Vitolo, E.: Individual and societal risk owing to landslides in the Campania region (Southern Italy), Georisk, 2, 125-140, 2008b.

CFDC (Centro Funzionale Decentrato della Campania): Centro Funzionale per la previsione meteorologica e il monitoraggio meteoidropluviometrico e delle frane, available at: http:// centrofunzionale.regione.campania.it/, last access: 23 November 2016.

Chirico, G. B., Di Crescenzo, G., Santangelo, N., Santo, A., and Scorpio, V.: Alluvial fan flooding hazard: the study case of Teglia (San Gregorio Magno, Salerno), Rend. Online Soc. Geol. It., 2, 456-458, 2012.

Coe, J. A., Godt, J. W., Parise, M., and Moscariello, A.: Estimating debris-flow probability using fan stratigraphy, historic records, and drainage-basin morphology, Interstate 70 highway corridor, central Colorado, U.S.A., in: Proc. 3rd International Conference 
on Debris-Flow Hazards Mitigation: Mechanics, Prediction, and Assessment, Davos (Switzerland), 2, 1085-1096, 2003.

Cossu, A., De Waele, J., and Di Gregorio, F.: Coastal karst geomorphosites at risk? A case study: the floods of 6-11 december 2004 in central-east Sardinia, in: Natural and Anthropogenic Hazards in Karst Areas: Recognition, Analysis, and Mitigation, edited by: Parise, M. and Gunn, J., Geological Society of London, special publ. 279, 85-95, 2007.

Costa, J. E.: Rheologic, Geomorphic and Sedimentologic differentiation of water floods, hyperconcentrated flows, and debris flows, Flood Geomorph., 113-122, 1988.

Crosta, G. B. and Dal Negro, P.: Observations and modelling of soil slip-debris flow initiation processes in pyroclastic deposits: the Sarno 1998 event, Nat. Hazards Earth Syst. Sci., 3, 53-69, doi:10.5194/nhess-3-53-2003, 2003.

Crosta, G. B. and Frattini, P.: Controls on modern Alluvial fan processes in the Central Alps, Northern Italy, Earth Surf. Proc. Land., 29, 267-293, 2004.

Delle Rose, M. and Parise, M.: Water management in the karst of Apulia, southern Italy, in: Proc. Int. Interdisc. Conf. "Sustainability of the karst environment. Dinaric karst and other karst regions", IHP-UNESCO, Series on Groundwater, 2, 33-40, 2010.

Del Prete, M., Guadagno, F. M., and Hawkins, A. B.: Preliminary report on the landslides of 5 May 1998, Campania, southern Italy, Bull. Eng. Geol. Environ., 57, 113-129, 1998.

Del Prete, S. and Mele, R.: Il contributo delle informazioni storiche per la valutazione della propensione al dissesto nell'Isola d'Ischia (Campania), Rend. Online Soc. Geol. It., 2, 29-47, 2006.

Di Crescenzo, G. and Santo, A.: Debris slides-rapid earth flows in the carbonate massifs of the Campania region (Southern Italy): Morphological and morphometric data for evaluating triggering susceptibility, Geomorphology, 66, 255-276, 2005.

Di Crescenzo, G., Liuzza, V., Santangelo, N., Santo, A., and Scorpio, V.: Flood susceptibility assessment in urbanized areas: cases study in Campanian Apennines, Mem. Descr. Carta Geol. It., 93, 203-218, 2013.

Esposito, G. and Galli, P.: La catastrofe idrogeologica del 1581 nei Monti Picentini (Sa) tra evidenze d'archivio e indagini geomorfologiche, Ital. J. Quat. Sci., 24, 179-189, 2011.

Esposito, E., Porfido, S., Violante, C., Molisso, F., Sacchi, M., Santoro, G., and Spiga, E.: Flood risk estimation through document sources analysis: the case of the Amalfi rocky coast, DTA, 2011.

Garfi, G., Bruno, D., Calcaterra, D., and Parise, M.: Fan morphodynamics and slope instability in the Mucone River basin (Sila Massif, southern Italy): significance of weathering and role of land use changes, Catena, 69, 181-196, 2007.

Gaume, E., Bain, V., Bernardara, P., Newinger, O., Barbuc, M., Bateman, A., Blaškovičová, L., Blöschl, G., Borga, M., Dumitrescu A., Daliakopoulos, I., Garcia, J., Irimescu, A., Kohnova, S., Koutroulis, A., Marchi, L., Matreata, S., Medina, V., Preciso, E., Sempere-Torres, D., Stancalie, G., Szolgay, J., Ioannis Tsanis, I., Velasco, D., and Viglione, D.: A compilation of data on European flash floods, J. Hydrol., 367, 70-78, 2009.

Gaume, E., Grunfest, E., Naulin, J.-P., Payrastre, O., and Vannier, O.: Social and hydrological responses to extrem precipitations: an interdisciplinary strategy for postflood investigation, Weather Clim. Soc., 6, 135-153, 2014.
Guadagno, F. M., Martino, S., and Mugnozza, G. S.: Influence of man-made cuts on the stability of pyroclastic covers (Campania, southern Italy): A numerical modelling approach, Environ. Geol., 43, 371-384, 2003.

Guadagno, F. M., Forte, R., Revellino, P., Fiorillo, F., and Focareta, M.: Some aspects of the initiation of debris avalanches in the Campania Region: The role of morphological slope discontinuities and the development of failure, Geomorphology, 66, 237254, 2005.

Guzzetti, F. and Tonelli, G.: Information system on hydrological and geomorphological catastrophes in Italy (SICI): a tool for managing landslide and flood hazards, Nat. Hazards Earth Syst. Sci., 4, 213-232, doi:10.5194/nhess-4-213-2004, 2004.

Guzzetti, F., Cardinali, M., and Reichenbach, P.: The AVI Project: a bibliographical and archive inventory of landslides and floods in Italy, Environ. Manage., 18, 623-633, 1994.

ISPRA-Servizio Geologico d'Italia: Progetto IFFI (Inventario dei Fenomeni Franosi in Italia), Landslide inventory map of Italy at 1:25000 scale, ISPRA - Dipartimento Difesa del Suolo-Servizio Geologico d'Italia - Regione Campania, available at http://193. 206.192.136/cartanetiffi/ (last access: 23 November 2016), 2006.

Iverson, R. M. and Vallance, J. W.: New views of granular mass flows, Geology, 29, 115-118, 2001.

Kirkham, R. M., Parise, M., and Cannon, S. H.: Geology of the 1994 South Canyon fire area, and a geomorphic analysis of the September 1, 1994 debris flows, south flank of Storm King Mountain, Glenwood Springs, Colorado, Colorado Geological Survey Special Publication, 46, 39 pp., 2000.

Komar, P. D.: Sediment transport by floods, in: Flood geomorphology, edited by: Baker, V. R., Kochel, R. C., and Patton, P. C., Wiley-INterscience, New York, 97-111, 1988.

Marchi, L. and Tecca, P. R.: Debris-flow monitoring in Italy, edited by: Schneuwly-Bollschweiler M., Stoffel M., and Rudolf-Miklau F., in: Dating Torrential Processes on Fans and Cones, Adv. Glob. Change Res., 47, 309-318, doi:10.1007/978-94-007-4336-620, 2013.

Marchi, L., Borga, M., Preciso, E., and Gaume, E.: Characterisation of selected extreme flash floods in Europe and implications for flood risk management, J. Hydrol., 394, 118-133, 2010.

Merheb, M., Moussa, R., Abdallah, C., Colin, F., Perrin, C., and Baghdadi, N.: Hydrological response characteristics of Mediterranean catchments at different time scales: a meta-analysis, Hydrolog. Sci. J., 61, 2520-2539, doi:10.1080/02626667.2016.114017, 2016.

Migale, L. S. and Milone, A.: Mud flows in pyroclastic deposits of the Campania, First data from historical research, Rassegna Storica Salernitana, 30, 15, 235-271, 1998.

Montz, B. E. and Gruntfest, E.: Flash flood mitigation: Recommendations for research and applications, Environ. Haz., 4, 15-22, 2002.

Palma, B., Calcaterra, D., and Parise, M.: Modelli geologici e meccanismi di innesco di frane da scorrimento-colata rapida nei depositi vulcanoclastici della Campania, GEAM, 126, 21-48, 2009.

Parise, M.: Flood history in the karst environment of CastellanaGrotte (Apulia, southern Italy), Nat. Hazards Earth Syst. Sci., 3, 593-604, doi:10.5194/nhess-3-593-2003, 2003.

Perrin, J. L. and Tournoud, M. G.: Hydrological processes controlling flow generation in a small Mediterranean catchment under karstic influence, Hydrolog. Sci. J., 54, 1125-1140, 2009. 
Pierson, T. C.: Distinguishing between debris flows and floods from field evidence in small watersheds (No. 2004-3142), US Geological Survey, 4 pp., 2005.

Pierson, T. C. and Costa, J. E: A rheologic classification of subaerial sediment-water flows, in: Geological Society of America Reviews in Eng. Geol., 7, 1-12, 1987.

Porfido, S., Esposito, E., Molisso, F., Sacchi, M., and Violante, C.: Flood Historical Data for Flood Risk Estimation in Coastal Areas, Eastern Tyrrhenian Sea, Italy, edited by: Margottini, C., Canuti, P., and Sassa, K., Landslide Science and Practice, 5, Springer-Verlag, Berlin Heidelberg, doi:10.1007/978-3-64231427-8_13, 2013.

Revellino, P., Hungr, O., Guadagno, F. M., and Evans, S. G.: Velocity and runout simulation of destructive debris flows and debris avalanches in pyroclastic deposits, Campania region, Italy, Environ. Geol., 45, 295-311, 2004.

Romano, P., Santo, A., and Voltaggio, M.: L'evoluzione geomorfologica della pianura del F. Volturno (Campania) durante il tardo Quaternario (Pleistocene medio-superiore - Olocene), Il Quaternario, 7, 41-56, 1994.

Ruiz-Villanueva, V., Díez-Herrero, A., Stoffel, M., Bollschweiler, M., Bodoque, J. M., and Ballesteros, J. A.: Dendrogeomorphic analysis of flash floods in a small ungauged mountain catchment (Central Spain), Geomorphology, 118, 383-392, 2010.

Salvati, P., Bianchi, C., Rossi, M., and Guzzetti, F.: Societal landslide and flood risk in Italy, Nat. Hazards Earth Syst. Sci., 10, 465-483, doi:10.5194/nhess-10-465-2010, 2010.

Santangelo, N., Santo, A. and Faillace, P.: Valutazione della pericolosità alluvionale delle conoidi del Vallo di Diano (Salerno, Italia meridionale), IJQS, 19, 3-17, 2006.

Santangelo, N., Santo, A., Di Crescenzo, G., Foscari, G., Liuzza, V., Sciarrotta, S., and Scorpio, V.: Flood susceptibility assessment in a highly urbanized alluvial fan: the case study of Sala Consilina (southern Italy), Nat. Hazards Earth Syst. Sci., 11, 2765-2780, doi:10.5194/nhess-11-2765-2011, 2011.

Santangelo, N., Daunis-i-Estadella, J., Di Crescenzo, G., Di Donato, V., Faillace, P., Martin-Fernandez, J. A., Romano, P., Santo, A., and Scorpio, V.: Topographic predictors of susceptibility to alluvial fan flooding, Southern Apennines, Earth Surf. Proc. Land., 37, 803-817, doi:10.1002/esp.3197, 2012.

Santo, A., Santangelo, N., Benedice, A., and Iovane, F.: Pericolosità connessa a processi alluvionali in aree pedemontane: il caso di Castellamare di Stabia in Penisola Sorrentina, Il Quaternario, 15, 23-37, 2002.
Santo, A., Di Crescenzo, G., Del Prete, S., and Di Iorio, L.: The Ischia island flash flood of November 2009 (Italy): Phenomenon analysis and flood hazard, Phys. Chem. Earth, 49, 3-17, 2012.

Santo, A., Santangelo, N., Di Crescenzo, G., Scorpio, V., De Falco, M., and Chirico, G. B.: Flash flood occurrence and magnitude assessment in an alluvial fan context: the October 2011 event in the Southern Apennines, Nat. Hazards, 78, 417-442, 2015.

Scorpio, V.: Analisi Geomorfologica dei sistemi bacino-conoide dell'Appennino campano: scenari di suscettibilità alluvionale, PhD Thesis, University of Napoli Federico II, 2011.

SIMN (Servizio Idrografico e Mareografico Nazionale): 1919-1996, Hydrological Annals, Part I, Naples, available at http://centrofunzionale.regione.campania.it/index.php/ documenti/annali, last access: 23 November 2016.

Simoni, A., Mammoliti, M., and Berti, M.: Uncertainty of debris flow mobility relationships and its influence on the prediction of inundated areas, Geomorphology, 132, 249-259, 2011.

SorrisoValvo, M., Antronico, L., and La Pera, E.: Controls on fan morphology in Calabria, southern Italy, Geomorphology, 24, 169-187, 1998.

Sosio, R., Crosta, G. B., and Frattini, P.: Field observations, rheological testing and numerical modelling of a debris-flow event, Earth Surf. Proc. Land., 32, 290-306, 2007.

Tiranti, D., Cremonini, R., Marco, F., Gaeta, A. R., and Barbero, S.: The DEFENSE (debris Flows triggered by storms-nowcasting system): An early warning system for torrential processes by radar storm tracking using a Geographic Information System (GIS), Comput. Geosci., 70, 96-109, 2014.

Tropeano, D. and Turconi, L.: Using historical documents for landslide, debris flow and stream flood prevention, Applications in Northern Italy, Nat. Hazards, 31, 663-679, 2004.

Vallario, A.: Il dissesto idrogeologico in Campania, CUEN, 2001.

Zanchetta, G., Sulpizio, R., and Di Vito, M. A.: The role of volcanic activity and climate in alluvial fan growth at volcanic areas: an example from Southern Campania (Italy), Sed. Geol., 168, 249280, 2004a.

Zanchetta, G., Sulpizio, R., Pareschi, M. T., Leoni, F. M., and Santacroce, R.: Document Characteristics of May 5-6, 1998 volcaniclastic debris flows in the Sarno area (Campania, southern Italy): Relationships to structural damage and hazard zonation, J. Volcanol. Geoth. Res., 133, 377-393, 2004 b. 